\title{
Implicit shock tracking using an optimization-based high-order discontinuous Galerkin method
}

\author{
M. J. Zahra, ${ }^{\mathrm{a}, *}$, A. Shi ${ }^{\mathrm{b}, 2}$, P.-O. Persson ${ }^{\mathrm{b}, \mathrm{c}, 3}$ \\ ${ }^{a}$ Department of Aerospace and Mechanical Engineering, University of Notre Dame, Notre Dame, IN 46556, United States \\ ${ }^{b}$ Department of Mathematics, University of California, Berkeley, Berkeley, CA 94720, United States \\ ${ }^{c}$ Mathematics Group, Lawrence Berkeley National Laboratory, 1 Cyclotron Road, Berkeley, CA 94720, United States
}

\begin{abstract}
A novel framework for resolving discontinuous solutions of conservation laws, e.g., contact lines, shock waves, and interfaces, using implicit tracking and a high-order discontinuous Galerkin (DG) discretization was introduced in [39]. Central to the framework is an optimization problem whose solution is a discontinuityaligned mesh and the corresponding high-order approximation to the flow that does not require explicit meshing of the unknown discontinuity surface. The method was shown to deliver highly accurate solutions on coarse, high-order discretizations without nonlinear stabilization and recover optimal convergence rates $\mathcal{O}\left(h^{p+1}\right)$ even for problems with discontinuous solutions. This work extends the implicit tracking framework such that robustness is improved and convergence accelerated. In particular, we introduce an improved formulation of the central optimization problem and an associated sequential quadratic programming (SQP) solver. The new error-based objective function penalizes violation of the DG residual in an enriched test space and is shown to have excellent tracking properties. The SQP solver simultaneously converges the nodal coordinates of the mesh and DG solution to their optimal values and is equipped with a number of features to ensure robust, fast convergence: Levenberg-Marquardt approximation of the Hessian with weighted elliptic regularization, backtracking line search based on the $\ell_{1}$ merit function, and rigorous convergence criteria. We use the proposed method to solve a range of inviscid conservation laws of varying difficulty. We show the method is able to deliver accurate solutions on coarse, high-order meshes and the SQP solver is robust and usually able to drive the first-order optimality system to tight tolerances.
\end{abstract}

Keywords: shock tracking, shock fitting, $r$-adaptivity, high-order methods, discontinuous Galerkin, high-speed flows

\section{Introduction}

High-order methods, such as the discontinuous Galerkin (DG) method [7, 19], are widely believed to be superior to traditional low-order schemes for simulation of turbulent flow problems. However, in the presence of shocks and other discontinuities such as contact lines or interfaces, the lack of nonlinear stability proves to be a fundamental challenge. These features are ubiquitous in engineering and science applications, particularly in high-speed flow problems. Many solutions have been proposed, but most require excessively refined meshes to resolve all the features, which in practices makes it difficult to accurately predict high Reynolds, high Mach flows that feature shocks, boundary layers, and interactions between them. Therefore, new advances are required to make high-order schemes sufficiently robust and competitive for real-world problems.

Most of the techniques for addressing shocks are based on so-called shock capturing, that is, the numerical discretization somehow incorporates the discontinuities independently of the computational grid. One simple

\footnotetext{
* Corresponding author

Email addresses: mzahr@nd.edu (M. J. Zahr), andrewshi94@berkeley.edu (A. Shi), persson@berkeley.edu (P.-O. Persson)

${ }^{1}$ Assistant Professor, Department of Aerospace and Mechanical Engineering, University of Notre Dame

${ }^{2}$ Graduate student, Department of Mathematics, University of California, Berkeley

${ }^{3}$ Professor, Department of Mathematics, University of California, Berkeley
} 
method is to use a sensor that identifies the mesh elements that contain shocks, and reduce their polynomial degrees $[3,6]$. For the DG method, this essentially leads to a standard cell-centered finite volume scheme locally, which is well-known to handle shocks robustly. Related, more sophisticated approaches include limiting, such as the weighted essentially non-oscillatory (WENO) schemes [16, 24, 20]. For high-order methods, artificial viscosity has also proven to be highly competitive, since it can smoothly resolve the jumps in the solution without introducing additional discontinuities between the elements [29]. The main problem with all these approaches is that they reduce to first order accuracy in the affected elements, which translates into a globally first order accurate scheme. This can be remedied by using local mesh refinement around the shock ( $h$-adaptivity) [12], although the anisotropic elements that are required for efficiency are difficult to generate and excessively fine elements are needed around the shock.

An alternative approach is shock tracking or shock fitting, where the computational mesh is moved such that their faces are aligned with the discontinuities in the solution [33, 34, 4, 17, 38, 37, 41, 35, 2, 31, 15, 28]. This is very natural in the setting of a DG method since the numerical scheme already incorporates jumps between the elements and the approximate Riemann solvers employed on the element faces handle the discontinuities correctly. However, it is a difficult meshing problem since it essentially requires generating a fitted mesh to the (unknown) shock surface. Also, in the early approaches to shock fitting, it was applied to low-order schemes where the relative advantage over shock capturing is smaller than for high-order methods. For these reasons, shock tracking is largely not used in practical CFD today.

In [39], we introduced a novel approach to shock tracking that does not require explicitly generating a mesh of the unknown discontinuity surface. Rather, the conservation law is discretized on a mesh without knowledge of the discontinuity surface and an optimization problem is formulated such that its solution is a mesh that aligns with discontinuities in the flow and the corresponding solution of the discrete conservation law. That is, tracking of the discontinuities is implicitly defined through the solution of the optimization problem and will be referred to as implicit shock tracking. While this approach works with any discretization that allows for inter-element discontinuities, we focus on high-order DG methods due to the high degree of accuracy attainable on coarse meshes, proper treatment of discontinuities with approximate Riemann solvers, and the ability to used curved elements to track discontinuities with curvature. The optimization problem is solved by simultaneously converging the mesh and solution to their optimal values, which never requires the fully converged DG solution on non-aligned meshes and does not require nonlinear stabilization. The combination of implicit tracking with a DG discretization is truly high-order accurate, since the solution is smooth within each element, and very accurate solutions can be obtained on coarse meshes.

This paper extends our prior work with a new error-based objective function, a sequential quadratic programming solver for the optimization problem, and a number of practical considerations. The proposed objective function penalizes violation of the DG residual in an enriched test space, which is a surrogate for violation of the weak formulation of the conservation law. Even though a traditional DG solution will oscillate about discontinuities in the solution on a non-aligned mesh, this violates the true conservation law; as a result, the proposed objective function promotes alignment of the mesh with discontinuities. This formulation has the added benefit of $r$-adaptive behavior; even in smooth regions of the flow, nodes will adjust to improve the approximation of the conservation law. The other main contribution of this work is an SQP solver for the optimization problem that leverages its structure. Due to the minimum-residual structure of the objective function, we employ a Levenberg-Marquardt approximation of the Hessian. We propose to use the stiffness matrix of a linear elliptic partial differential equation (PDE) with the coefficient chosen inversely proportional to the local element size as the regularization matrix. This tends to smooth out the search directions for the mesh coordinates and is particularly important for problems with elements of significantly different size. The SQP method is globalized with a line search based on the $\ell_{1}$ merit function and equipped with termination conditions based on the first-order optimality criteria. For the method to be practical for difficult problems, we initialize the solve with the $p=0$ DG solution and use continuation in the polynomial degree (solution and mesh) for high-order $(p>1)$ discretizations. Finally, we identified the critical role of smoothness of the DG numerical flux function with respect to variations in the element normal in the context of implicit shock tracking, mainly with respect to solver convergence, and use smoothed versions of traditional numerical fluxes throughout.

To our knowledge, the only other approach to implicit shock tracking was proposed in $[10,11,8,9,22]$. where the authors enforce a DG discretization with unconventional numerical fluxes and the RankineHugoniot interface conditions in a minimum-residual sense. Interestingly, enforcement of the interface 
condition circumvented traditional stability requirements for the DG numerical fluxes, allowing them to solely rely on fluxes interior to an element. Their method was shown to successfully track even complex discontinuity surfaces and provide accurate approximations to the conservation law on traditionally coarse, high-order meshes. The present work incorporates some aspects of their method, in particular topological mesh operations and some aspects of the Hessian approximation and regularization. However, our approach that directly enforces a stable DG discretization (with zero residual) inherits many attractive features of DG methods such as guaranteed conservation and a rigorous framework for high-order convergence. Furthermore, by choosing an error-based objective function rather than a physics-based one, the extension to viscous problems only requires treatment of second-order terms in the DG setting, which has been well-established [1].

The remainder of the paper is organized as follows. Section 2 introduces the governing system of inviscid conservation laws and its discretization using a discontinuous Galerkin method. Section 3 recalls the implicit tracking framework originally proposed in [39], introduces the new error-based objective function, and discusses a parametrization of the mesh deformation that ensures the boundaries of the computational domain remain on the boundaries of the actual domain. Section 4 introduces the proposed SQP solver for the central optimization problem that incorporates a Levenberg-Marquardt approximation of the Hessian with a novel regularization matrix, a line search based on a $\ell_{1}$ merit function, and termination criteria based on the first-order optimality conditions. Section 5 discusses two important details required to make the proposed tracking framework work in practice: initialization of the SQP solver and topological mesh operations to remove small elements. Finally, Section 6 presents a number of numerical experiments that demonstrate the method is able to accurately approximate complex flows using coarse, high-order meshes and the SQP solver is able to quickly converge to a mesh that tracks all discontinuities and exhibits deep convergence to the first-order optimality conditions.

\section{Governing equations and high-order numerical discretization}

Consider a general system of $M$ inviscid conservation laws, defined on the physical domain $\Omega \subset \mathbb{R}^{d}$ and subject to appropriate boundary conditions,

$$
\nabla \cdot F(U)=S(U) \text { in } \Omega
$$

where $U: \Omega \rightarrow \mathbb{R}^{M}$ is the solution of the system of conservation laws, $F: \mathbb{R}^{M} \rightarrow \mathbb{R}^{M \times d}$ is the physical flux, $S: \mathbb{R}^{M} \rightarrow \mathbb{R}^{M}$ is the source term, and $\nabla:=\left(\partial_{x_{1}}, \ldots, \partial_{x_{d}}\right)$ is the gradient operator in the physical domain such that $\nabla w(x)=\left[\begin{array}{llll}\partial_{x_{1}} w(x) & \cdots & \partial_{x_{d}} w(x)\end{array}\right] \in \mathbb{R}^{N \times d}$ for any $N$ vector-valued function $w$ over $\Omega$ $\left(w(x) \in \mathbb{R}^{N}\right.$ for $\left.x \in \Omega\right)$. The boundary of the domain is $\partial \Omega$ with outward unit normal $n: \partial \Omega \rightarrow \mathbb{R}^{d}$. The formulation of the conservation law in (1) is sufficiently general to encapsulate steady conservation laws in a $d$-dimensional spatial domain or time-dependent conservation laws in a $(d-1)$-dimensional domain, i.e., a $d$-dimensional space-time domain. In general, the solution $U(x)$ may contain discontinuities, in which case, the conservation law (1) holds away from the discontinuities and the Rankine-Hugoniot conditions [26]

$$
F\left(U^{+}\right) n=F\left(U^{-}\right) n
$$

hold for $x \in \Gamma_{s}$, where $\Gamma_{s} \subset \Omega$ is a surface along which $U$ is discontinuous, $U^{+}(x), U^{-}(x) \in \mathbb{R}^{M}$ are the values of $U(x)$ on either side of the discontinuity, and $n$ is a normal vector to the surface $\Gamma_{s}$.

Similar to our previous work [39], we will construct a numerical method that directly tracks discontinuities with the computational grid, which places three requirements on the discretization: 1) represents a stable and convergent discretization of the conservation law in (1), 2) allows for deformation of the computational domain, and 3) employs a solution basis that supports discontinuities between computational cells or elements. To achieve high-order accuracy, the tracking framework introduced in [39] is built upon a standard high-order DG method given their proven ability $[39,8]$ to deliver accurate solutions on very coarse discretizations provided discontinuities are tracked. The extension to other discretizations that support inter-element discontinuities such as finite volumes, flux reconstruction, hybridizable DG, and the DG spectral element method are possible, but beyond the scope of this work. 
The remainder of this section will detail the discretization of the conservation law (1) using DG such that it reduces to the discrete form: given $\boldsymbol{x} \in \mathbb{R}^{N_{\boldsymbol{x}}}$, find $\boldsymbol{u} \in \mathbb{R}^{N_{u}}$

$$
\boldsymbol{r}(\boldsymbol{u}, \boldsymbol{x})=\mathbf{0}
$$

where $\boldsymbol{u}$ is the discrete representation of the conservation law state $U, \boldsymbol{x}$ is the discrete representation of the conservation law domain $\Omega$ (nodal coordinates of mesh nodes), and $\boldsymbol{r}: \mathbb{R}^{N_{u}} \times \mathbb{R}^{N_{x}} \rightarrow \mathbb{R}^{N_{u}}$ is the discretized conservation law. The same discretization will be used to define a residual function based on an enriched test space $\boldsymbol{R}: \mathbb{R}^{N_{u}} \times \mathbb{R}^{N_{\boldsymbol{x}}} \rightarrow \mathbb{R}^{N_{u}}$, which will be used in the new definition of the proposed objective function.

\subsection{Transformed conservation law from deformation of physical domain}

Before introducing a discretization of (1) it is convenient to explicitly treat deformations to the domain of the conservation law $\Omega$, which will eventually be induced by deformation to the mesh as nodal coordinates are moved to track discontinuities, by transforming to a fixed reference domain $\Omega_{0} \subset \mathbb{R}^{d}$. Suppose the physical domain can be taken as the result of a diffeomorphism applied to a reference domain (Figure 1)

$$
\Omega=\mathcal{G}\left(\Omega_{0}\right),
$$

where $\Omega_{0} \subset \mathbb{R}^{d}$ is a fixed reference domain and $\mathcal{G}: \mathbb{R}^{d} \rightarrow \mathbb{R}^{d}$ is the diffeomorphism defining the domain mapping.

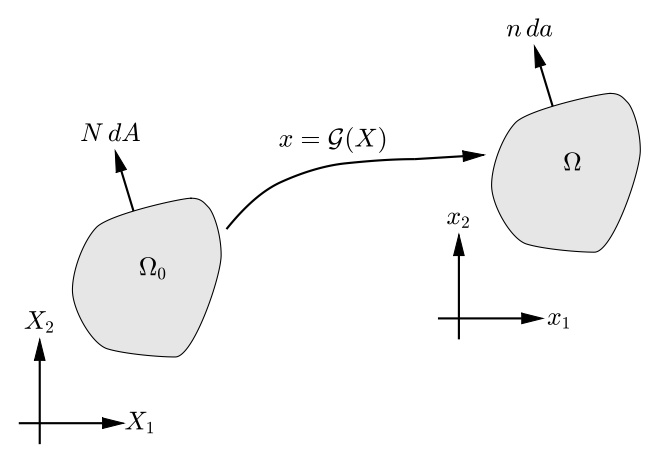

Figure 1: Mapping between reference and physical domains.

For convenience, the conservation law on the physical domain $\Omega$ is transformed to a conservation law on the reference domain

$$
\nabla_{X} \cdot F_{X}\left(U_{X}, \mathcal{G}\right)=S_{X}\left(U_{X}, \mathcal{G}\right) \text { in } \Omega_{0}
$$

where $\nabla_{X}:=\left(\partial_{X_{1}}, \ldots, \partial_{X_{d}}\right)$ denotes spatial derivatives with respect to the reference domain $\Omega_{0}$ with coordinates $X, U_{X}: \Omega_{0} \rightarrow \mathbb{R}^{M}$ is the mapped state vector we define as

$$
U_{X}=U \circ \mathcal{G}
$$

$F_{X}\left(U_{X}, \mathcal{G}\right) \in \mathbb{R}^{M \times d}$ is the transformed flux function, and $S_{X}\left(U_{X}, \mathcal{G}\right) \in \mathbb{R}^{M}$ is the transformed source term. The unit normal in the reference and physical domain are related by

$$
n=\frac{g G^{-T} N}{\left\|g G^{-T} N\right\|}
$$

where $G(X)=\frac{\partial}{\partial X} \mathcal{G}(X)$ is the deformation gradient of the domain mapping and $g(X)=\operatorname{det} G(X)$ is the Jacobian.

For the transformed conservation law (5) and original conservation law (1) to be equivalent, we require

$$
\int_{V}\left(\nabla_{X} \cdot F_{X}-S_{X}\right) d V=\int_{\mathcal{G}(V)}(\nabla \cdot F-S) d v
$$


holds over an arbitrary volume $V \subset \Omega_{0}$ and arguments have been dropped for convenience. Considering the source term and flux individually,

$$
\int_{\mathcal{G}(V)} S d v=\int_{V} g S d V
$$

follows directly from a change of variables in the integral and

$$
\int_{\mathcal{G}(V)} \nabla \cdot F d v=\int_{\partial \mathcal{G}(V)} F \cdot n d s=\int_{\partial V} g F \cdot G^{-T} N d S=\int_{V} \nabla_{X} \cdot\left(g F \cdot G^{-T}\right) d S .
$$

follows from the divergence theorem in the physical domain, change of variables for surface integrals (Nanson's formula), and the divergence theorem in the reference domain. Combining (8)-(10) and invoking arbitrariness of the volume $V$, the transformed flux and source term take the following form

$$
F_{X}\left(U_{X}, \mathcal{G}\right)=g F(U) G^{-T}, \quad S_{X}\left(U_{X}, \mathcal{G}\right)=g S(U) .
$$

\subsection{Discontinuous Galerkin discretization of transformed conservation law}

We use a standard nodal discontinuous Galerkin method $[7,18]$ to discretize the transformed conservation law (5). Let $\mathcal{E}_{h, q}$ represent a discretization of the reference domain $\Omega_{0}$ into non-overlapping, potentially curved, computational elements, where $h$ is a mesh element size parameter and $q$ is the polynomial order associated with the curved elements. The DG construction begins with the elementwise weak form of the conservation law (5) that results from multiplying each equation by a test function $\psi_{X}$, integrating over a single element $K \in \mathcal{E}_{h, q}$, and applying the divergence theorem

$$
\int_{\partial K} \psi_{X}^{+} \cdot F_{X}\left(U_{X}, \mathcal{G}\right) N d S-\int_{K} F\left(U_{X}, \mathcal{G}\right): \nabla_{X} \psi_{X} d V=0,
$$

where $N$ is the outward normal to the surface $\partial K$ and $\psi_{X}^{+}$denotes the trace of $\psi$ interior to element $K$. To ensure the face integrals are single-valued, we replace $F_{X}\left(U_{X}, \mathcal{G}\right) N$ in the first term with a numerical flux function $\mathcal{H}_{X}\left(U_{X}^{+}, U_{X}^{-}, N, \mathcal{G}\right)$

$$
\int_{\partial K} \psi_{X}^{+} \cdot \mathcal{H}_{X}\left(U_{X}^{+}, U_{X}^{-}, N, \mathcal{G}\right) d S-\int_{K} F\left(U_{X}, \mathcal{G}\right): \nabla_{X} \psi_{X} d V=0,
$$

where $U_{X}^{+}$denotes the interior trace of $U_{X}$ and $U_{X}^{-}$denotes the exterior trace of $U_{X}$ if $\partial K$ is an interior face, i.e., $\partial K \cap \partial \Omega_{0}=\varnothing$, otherwise $U_{X}^{-}$is a boundary state $U_{X}^{\partial}\left(U_{X}, n\right)$ constructed to enforce the appropriate boundary condition. We defer a detailed discussion of numerical flux functions to Section 2.3 and boundary conditions to the specific conservation laws considered in Section 6.

To establish the finite-dimensional form of (13), we introduce the mapped finite element space of piecewise polynomial functions associated with the mesh $\mathcal{E}_{h, q}$ :

$$
\mathcal{V}_{h, p}=\left\{v \in\left[L^{2}\left(\Omega_{0}\right)\right]^{M}|v|_{K} \circ \mathcal{T}_{K} \in\left[\mathcal{P}_{p}\left(K_{0}\right)\right]^{M} \forall K \in \mathcal{E}_{h, q}\right\}
$$

where $\mathcal{P}_{p}\left(K_{0}\right)$ is the space of polynomial functions of degree at most $p \geqslant 1$ on the parent element $K_{0}$ and $K=\mathcal{T}_{K}\left(K_{0}\right)$ defines a mapping from the parent element to element $K \in \mathcal{E}_{h, q}$. For notational brevity, we assume all elements map from a single parent element. The finite-dimensional residual of the weak form in (13) corresponding to the trial space $\mathcal{V}_{h, p}$ and test space $\mathcal{V}_{h^{\prime}, p^{\prime}}$ is

$$
r_{X_{h^{\prime}, p^{\prime}}}^{K}\left(U_{X_{h, p}}, \mathcal{G}\right):=\int_{\partial K} \psi_{X_{h^{\prime}, p^{\prime}}^{+}}^{+} \cdot \mathcal{H}_{X}\left(U_{X_{h, p}}^{+}, U_{X_{h, p}}^{-}, N, \mathcal{G}\right) d S-\int_{K} F_{X}\left(U_{X_{h, p}}, \mathcal{G}\right): \nabla_{X} \psi_{X_{h^{\prime}, p^{\prime}}} d V
$$

Finally, define the continuous global function space

$$
\mathcal{W}_{h, q}:=\mathcal{V}_{h, q} \cap C^{0}(\Omega),
$$

and constrain domain deformation to lie in it: $\mathcal{G} \approx \mathcal{G}_{h, q} \in \mathcal{W}_{h, q}$. After summing over all elements $K \in \mathcal{E}_{h, q}$, the final version of the finite-dimensional Galerkin weak form is: given $\mathcal{G}_{h, q} \in \mathcal{W}_{h, q}$, find $U_{X_{h, p}} \in \mathcal{V}_{h, p}$ such 
that

$$
\sum_{K \in \mathcal{E}_{h, q}} r_{X_{h, p}}^{K}\left(U_{X_{h, p}}, \mathcal{G}_{h, q}\right)=0
$$

for all $\psi_{X_{h, p}} \in \mathcal{V}_{h, p}$.

To establish the discrete (algebraic) form of (16), we introduce a (nodal) basis over each element and expand the finite-dimensional test functions $\left(\psi_{X_{h, p}}\right)$, solution $\left(U_{X_{h, p}}\right)$, and domain deformation $\left(\mathcal{G}_{h, q}\right)$ in terms of these basis functions and coefficients. Invoking arbitrariness of the test functions in $\mathcal{V}_{h, p}$ and assembling an algebraic system that respects the global functions spaces in (15), we obtain

$$
\boldsymbol{r}(\boldsymbol{u}, \boldsymbol{x})=\mathbf{0},
$$

where $\boldsymbol{u} \in \mathbb{R}^{N_{u}}$ are the (assembled) coefficients of the solution $U_{X_{h, p}} \in \mathcal{V}_{h, p}$ and $\boldsymbol{x} \in \mathbb{R}^{N_{\boldsymbol{x}}}$ are the (assembled) coefficients of the domain deformation $\mathcal{G}_{h, q} \in \mathcal{W}_{h, q}$. Since we are using nodal bases, the entries of $\boldsymbol{x}$ are the coordinates of the nodes of the mesh and $\boldsymbol{u}$ contains the components of the solution at the nodes.

To close this section, we introduce an enriched discrete residual $\boldsymbol{R}(\boldsymbol{u}, \boldsymbol{x})$ that will be used to define the proposed shock tracking objective function. Let $\boldsymbol{R}(\boldsymbol{u}, \boldsymbol{x})$ be the algebraic version of

$$
\sum_{K \in \mathcal{E}_{h, q}} r_{X_{h^{\prime}, p^{\prime}}}^{K}\left(U_{X_{h, p}}, \mathcal{G}_{h, q}\right)
$$

where $h^{\prime} \leqslant h$ and $p^{\prime} \geqslant p$. That is, the enriched residual is defined by the same trial space $\left(\mathcal{V}_{h, p}\right)$ and space for the domain deformation $\left(\mathcal{W}_{h, q}\right)$ as the residual in (17), but uses an enriched test space $\mathcal{V}_{h^{\prime}, p^{\prime}}$. In this work, we take $h^{\prime}=h$ and $p^{\prime}=p+1$ although other choices are possible and will be explored in future work.

\subsection{Numerical flux function}

Recall the numerical flux is a quantity that replaces the flux dotted with the outward unit normal and expect it to transform according to

$$
\mathcal{H}_{X}\left(U_{X}^{+}, U_{X}^{-}, N, \mathcal{G}\right)=\left\|g G^{-T} N\right\| \mathcal{H}\left(U^{+}, U^{-}, n\right),
$$

which follows from (7) and (11) as

$$
\mathcal{H}_{X} \sim F_{X} \cdot N=g F \cdot G^{-T} N=\left\|g G^{-T} N\right\| F \cdot n \sim\left\|g G^{-T} N\right\| \mathcal{H},
$$

where arguments have been dropped for brevity. Therefore, the mapped numerical flux is uniquely determined from the physical numerical flux. For the remainder of this section, we will discuss the numerical flux in the physical domain $\mathcal{H}\left(U^{+}, U^{-}, n\right)$ and it will be transformed to the reference domain according to (19).

For DG methods to be stable, the numerical flux may be any two-point monotone Lipschitz function that is

(i) consistent with the flux function, i.e., for any $U \in \mathbb{R}^{M}$

$$
\mathcal{H}(U, U, n)=F(U) \cdot n
$$

(ii) conservative, i.e., for any $U, U^{\prime} \in \mathbb{R}^{M}$

$$
\mathcal{H}\left(U, U^{\prime}, n\right)=-\mathcal{H}\left(U^{\prime}, U,-n\right) .
$$

Conditions (i)-(ii) are satisfied by all standard numerical fluxes as these are the minimum requirements for a stable and accurate DG method. However, it was observed in $[39,8]$ that the requirements are higher for tracking-based discretizations since inter-element jumps do not tend to zero under refinement. As introduced in [8], this requires the additional condition:

(iii) preservation of the Rankine-Hugoniot conditions, i.e., given $U^{+}, U^{-} \in \mathbb{R}^{M}$ such that $F\left(U^{+}\right) \cdot n=$ $F\left(U^{-}\right) \cdot n$, then

$$
\mathcal{H}\left(U^{+}, U^{-}, n\right)=F\left(U^{+}\right) \cdot n=F\left(U^{-}\right) \cdot n
$$


Conditions (i)-(iii) are satisfied by exact Riemann solvers and a number of approximate Riemann solvers including the Roe [32] and HLLC [36] fluxes; however, many popular numerical flux functions such as the local Lax-Friedrichs, Rusanov, and Roe with entropy fix do not satisfy (iii) [8]. We highlight one additional desired property of the numerical flux function that we will show is important for optimization-based discontinuity tracking:

(iv) smoothness with respect to variations in the normal.

Since the numerical flux must have upwind-like properties, it is difficult to construct numerical fluxes to satisfy (i)-(iv), particularly the smoothness property. Therefore, we choose numerical fluxes that satisfy (i)-(iii) and replace non-smooth terms with smooth approximations to recover smoothness (iv). Strictly speaking, this will not preserve the Rankine-Hugoniot conditions (iii); in Section 6 we demonstrate this is a desirable trade-off.

As an example, consider linear advection of a scalar field $u: \Omega \rightarrow \mathbb{R}$ in a spatially varying direction $\beta: \Omega \rightarrow \mathbb{R}^{d}$ governed by conservation law of the form (1) (see (73) in Section 6.1) with flux function

$$
F_{\text {adv }}(u ; \beta)=u \beta^{T}
$$

and the upwind numerical flux

$$
\mathcal{H}_{\text {up }}\left(u^{+}, u^{-}, n\right)= \begin{cases}(\beta \cdot n) u^{+} & \text {if } \beta \cdot n \geqslant 0 \\ (\beta \cdot n) u^{-} & \text {if } \beta \cdot n<0,\end{cases}
$$

which can equivalently be written in terms of the Heaviside function $H: \mathbb{R} \rightarrow\{0,1\}$ as

$$
\mathcal{H}_{\text {up }}\left(u^{+}, u^{-}, n\right)=(\beta \cdot n)\left[u^{+} \cdot H(\beta \cdot n)+u^{-} \cdot(1-H(\beta \cdot n))\right] .
$$

The upwind flux satisfies conditions (i)-(iii) for an admissible flux for shock tracking, but fails to satisfy condition (iv). Condition (i) follows from

$$
\mathcal{H}_{\text {up }}(u, u, n)=(\beta \cdot n) u=F_{\text {adv }}(u) n
$$

and condition (ii) follows from

$$
-\mathcal{H}\left(u^{\prime}, u,-n\right)=(\beta \cdot n)\left[u^{\prime} \cdot H(-\beta \cdot n)+u \cdot(1-H(-\beta \cdot n))\right]=\mathcal{H}\left(u, u^{\prime}, n\right),
$$

for any $u, u^{\prime} \in \mathbb{R}$, where the second equality follows from the property of the Heaviside function: $H(-s)=$ $1-H(s)$ for $s \in \mathbb{R}$. To verify condition (iii), consider $u^{+}, u^{-} \in \mathbb{R}$ such that $u^{+} \neq u^{-}$(discontinuity) and assume $F_{\text {adv }}\left(u^{+}\right) \cdot n=F_{\text {adv }}\left(u^{-}\right) \cdot n$. This condition implies $\left(u^{+}-u^{-}\right)(\beta \cdot n)=0$, which in turn implies $\beta \cdot n=0$ from the assumption that $u^{+} \neq u^{-}$. Therefore condition (iii) holds from

$$
\mathcal{H}\left(u^{+}, u^{-}, n\right)=F_{\text {adv }}\left(u^{+}\right) n=F_{\text {adv }}\left(u^{-}\right) n=0 .
$$

Finally, it is easy to see that condition (iv) is not satisfied; the upwind flux is continuous with respect to variations in the normal $n$, but not smooth due to the $H(\beta \cdot n)$ terms. A single isolated point of nonsmoothness does not necessarily hinder the optimization solver because it is unlikely to be visited during the solution procedure. However, in this case, the kinks in the numerical flux function lie at points where $\beta \cdot n=0$, which is precisely the requirement for a discontinuous solution to satisfy the Rankine-Hugoniot conditions and will certainly be approached as the mesh faces align with the discontinuity.

To recover condition (iv), we introduce a smoothed version of the upwind flux where the Heaviside function is replaced with a smoothed step function $H_{a}: \mathbb{R} \rightarrow \mathbb{R}$

$$
\mathcal{H}_{\mathrm{up}}^{a}\left(U^{+}, U^{-}, n\right)=(\beta \cdot n)\left[U^{+} \cdot H_{a}(\beta \cdot n)+U^{-} \cdot\left(1-H_{a}(\beta \cdot n)\right)\right] .
$$




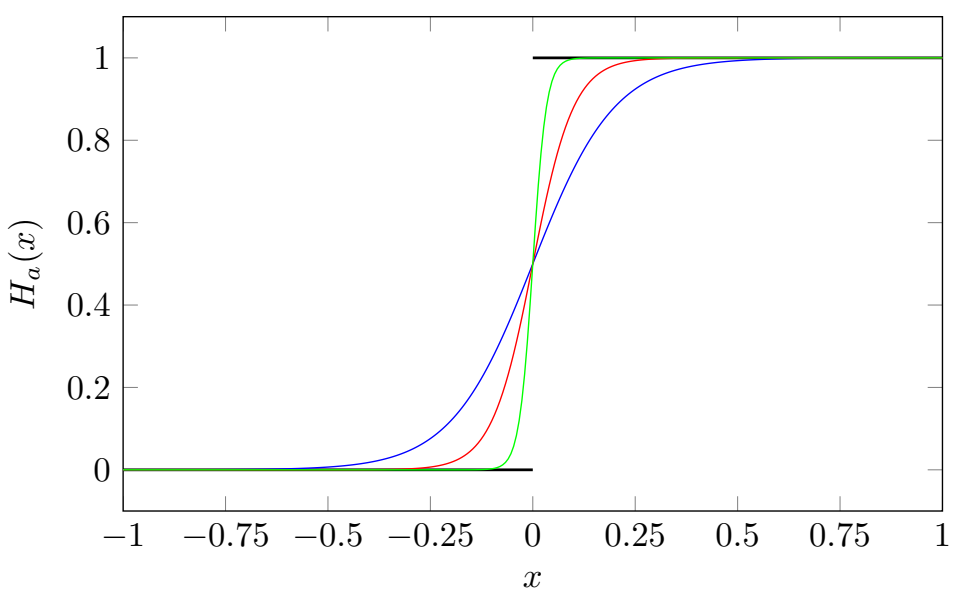

Figure 2: Smoothed Heaviside (logistic) function for $a=5(\square), a=10(\square), a=30(\square), a=\infty(\square)$.

In this work, we use the logistic function as the smoothed step function

$$
H_{a}(x):=\frac{1}{1+e^{-2 a x}},
$$

where $a \in \mathbb{R}$ is the smoothing parameter (Figure 2). Section 6 provides a detailed study of the impact of the smoothness of the numerical flux on the convergence of the tracking algorithm.

\section{Optimization formulation of $r$-adaptivity for implicit tracking of discontinuities}

In this section, we introduce the main contribution of this work: an $r$-adaptivity framework that recasts the discrete conservation law (17) as an optimization problem over the discrete solution and mesh that aims to align features in the solution basis with features in the solution itself. In this work these features are discontinuities since we only consider inviscid conservation laws; however, future work will consider steep gradients (viscous conservation laws) and interfaces. In the present setting, this amounts to aligning element faces with discontinuities. The method builds upon our previous work [39], where we demonstrated that high-order methods are capable of approximating discontinuous solutions of PDEs using extremely coarse discretizations provided the discontinuities are tracked. In this section, we focus on the new aspects of the optimization formulation and the next section will address the issue of solvers for the optimization problem. In particular, we introduce a new error-like objective function based on the DG residual using an enriched test space and a term to penalize mesh distortion.

\subsection{Constrained optimization formulation}

Following our work in [39], we formulate the problem of tracking discontinuities as a constrained optimization problem over the PDE state and coordinates of the mesh nodes that minimizes some objective function $f: \mathbb{R}^{N_{u}} \times \mathbb{R}^{N_{x}} \rightarrow \mathbb{R}$ while enforcing the DG discretization of the conservation law

$$
\begin{array}{ll}
\underset{\boldsymbol{u} \in \mathbb{R}^{N_{\boldsymbol{u}}}, \boldsymbol{x} \in \mathbb{R}^{N_{\boldsymbol{x}}}}{\operatorname{minimize}} & f(\boldsymbol{u}, \boldsymbol{x}) \\
\text { subject to } & \boldsymbol{r}(\boldsymbol{u}, \boldsymbol{x})=\mathbf{0} .
\end{array}
$$

The objective function is constructed such that the solution of the optimization problem is a mesh that aligns with discontinuities in the solution. The optimization-based tracking method directly inherits the benefits of standard DG methods, i.e., high-order accuracy and conservation, due to the constraint that exactly enforces the DG discretization. Finally, the optimization formulation in (32) will provide nonlinear stability if all discontinuities are successfully tracked, which we will demonstrate using several examples in Section 6. 
The Lagrangian of the optimization problem in (32) $\mathcal{L}: \mathbb{R}^{N_{u}} \times \mathbb{R}^{N_{\boldsymbol{x}}} \times \mathbb{R}^{N_{u}} \rightarrow \mathbb{R}$ takes the form

$$
\mathcal{L}(\boldsymbol{u}, \boldsymbol{x}, \boldsymbol{\lambda})=f(\boldsymbol{u}, \boldsymbol{x})-\boldsymbol{\lambda}^{T} \boldsymbol{r}(\boldsymbol{u}, \boldsymbol{x}),
$$

where $\boldsymbol{\lambda} \in \mathbb{R}^{N_{u}}$ is a vector of Lagrange multipliers associated with the DG constraint in (32). The first-order optimality, or Karush-Kuhn-Tucker (KKT), conditions state that the $\left(\boldsymbol{u}^{\star}, \boldsymbol{x}^{\star}\right)$ is a first-order solution of the optimization problem if there exists $\boldsymbol{\lambda}^{\star}$ such that

$$
\nabla_{\boldsymbol{u}} \mathcal{L}\left(\boldsymbol{u}^{\star}, \boldsymbol{x}^{\star}, \boldsymbol{\lambda}^{\star}\right)=\mathbf{0}, \quad \nabla_{\boldsymbol{x}} \mathcal{L}\left(\boldsymbol{u}^{\star}, \boldsymbol{x}^{\star}, \boldsymbol{\lambda}^{\star}\right)=\mathbf{0}, \quad \nabla_{\boldsymbol{\lambda}} \mathcal{L}\left(\boldsymbol{u}^{\star}, \boldsymbol{x}^{\star}, \boldsymbol{\lambda}^{\star}\right)=\mathbf{0},
$$

or equivalently,

$$
\frac{\partial f}{\partial \boldsymbol{u}}\left(\boldsymbol{u}^{\star}, \boldsymbol{x}^{\star}\right)^{T}-\frac{\partial \boldsymbol{r}}{\partial \boldsymbol{u}}\left(\boldsymbol{u}^{\star}, \boldsymbol{x}^{\star}\right)^{T} \boldsymbol{\lambda}^{\star}=\mathbf{0}, \quad \frac{\partial f}{\partial \boldsymbol{x}}\left(\boldsymbol{u}^{\star}, \boldsymbol{x}^{\star}\right)^{T}-\frac{\partial \boldsymbol{r}}{\partial \boldsymbol{x}}\left(\boldsymbol{u}^{\star}, \boldsymbol{x}^{\star}\right)^{T} \boldsymbol{\lambda}^{\star}=\mathbf{0}, \quad \boldsymbol{r}\left(\boldsymbol{u}^{\star}, \boldsymbol{x}^{\star}\right)=\mathbf{0} .
$$

Since the DG Jacobian with respect to the state variables $\boldsymbol{u}$ is assumed to be invertible, we define the estimate of the optimal Lagrange multiplier $\hat{\lambda}: \mathbb{R}^{N_{u}} \times \mathbb{R}^{N_{\boldsymbol{x}}} \rightarrow \mathbb{R}^{N_{u}}$ such that the first equation $\left(\nabla_{\boldsymbol{u}} \mathcal{L}=0\right)$ (adjoint equation) is always satisfied

$$
\hat{\boldsymbol{\lambda}}(\boldsymbol{u}, \boldsymbol{x})=\frac{\partial \boldsymbol{r}}{\partial \boldsymbol{u}}(\boldsymbol{u}, \boldsymbol{x})^{-T} \frac{\partial f}{\partial \boldsymbol{u}}(\boldsymbol{u}, \boldsymbol{x})^{T} .
$$

Then the optimality criteria becomes

$$
c\left(u^{\star}, x^{\star}\right)=0, \quad r\left(u^{\star}, x^{\star}\right)=0,
$$

where $c: \mathbb{R}^{N_{u}} \times \mathbb{R}^{N_{\boldsymbol{x}}} \rightarrow \mathbb{R}^{N_{u}}$ is defined as

$$
\boldsymbol{c}(\boldsymbol{u}, \boldsymbol{x}):=\nabla_{\boldsymbol{x}} \mathcal{L}(\boldsymbol{u}, \boldsymbol{x}, \hat{\boldsymbol{\lambda}}(\boldsymbol{u}, \boldsymbol{x}))=\frac{\partial f}{\partial \boldsymbol{x}}(\boldsymbol{u}, \boldsymbol{x})^{T}-\frac{\partial \boldsymbol{r}}{\partial \boldsymbol{x}}(\boldsymbol{u}, \boldsymbol{x})^{T} \frac{\partial \boldsymbol{r}}{\partial \boldsymbol{u}}(\boldsymbol{u}, \boldsymbol{x})^{-T} \frac{\partial f}{\partial \boldsymbol{u}}(\boldsymbol{u}, \boldsymbol{x})^{T} .
$$

In Section 4.7, $\|\boldsymbol{c}(\boldsymbol{u}, \boldsymbol{x})\|$ and $\|\boldsymbol{r}(\boldsymbol{u}, \boldsymbol{x})\|$ will be used to define the termination criteria for the proposed solver.

\subsection{Choice of objective function}

We propose an objective function that consists of two terms: one term penalizes a measure of the DG solution error $f_{\text {err }}: \mathbb{R}^{N_{u}} \times \mathbb{R}^{N_{x}} \rightarrow \mathbb{R}$ and the other term penalizes distortion of the mesh $f_{\mathrm{msh}}: \mathbb{R}^{N_{\boldsymbol{x}}} \rightarrow \mathbb{R}$, i.e.,

$$
f(\boldsymbol{u}, \boldsymbol{x})=f_{\mathrm{err}}(\boldsymbol{u}, \boldsymbol{x})+\kappa^{2} f_{\mathrm{msh}}(\boldsymbol{x}),
$$

where $\kappa \in \mathbb{R}_{+}$is a parameter that weights the contribution of the two terms. Since a piecewise polynomial solution on an aligned mesh will have much lower error than on a non-aligned mesh, $f_{\text {err }}$ promotes alignment of the mesh with discontinuities while $f_{\text {msh }}$ prevents the mesh from entangling or becoming unacceptably skewed.

For the error-like tracking term, we use the norm of the DG residual corresponding to an enriched test space, i.e.,

$$
f_{\mathrm{err}}(\boldsymbol{u}, \boldsymbol{x}):=\frac{1}{2} \boldsymbol{R}(\boldsymbol{u}, \boldsymbol{x})^{T} \boldsymbol{R}(\boldsymbol{u}, \boldsymbol{x}),
$$

where we enrich the test space using polynomials of one degree higher than the trial space. This follows on a large body of work that uses residual-based error indicators to drive $h-, p$-, and $r$-adaptivity [13]. This is a reasonable choice for the objective function because the enriched test space adds additional constraints to the solution and even though a discrete solution $\boldsymbol{u}$ containing significant oscillations satisfies $\boldsymbol{r}(\boldsymbol{u}, \boldsymbol{x})=\mathbf{0}$, it will likely not minimize $\boldsymbol{R}(\cdot, \boldsymbol{x})$. Furthermore, the more the test space is enriched, the closer the DG residual comes to enforcing the true conservation law and therefore the minimum-residual solution approaches the exact solution of the PDE. Given that we are explicitly enforcing the constraint $\boldsymbol{r}(\boldsymbol{u}, \boldsymbol{x})=\mathbf{0}$, which fixes $\boldsymbol{u}$ for a given $\boldsymbol{x}$ (assuming $\boldsymbol{r}(\cdot, \boldsymbol{x})=\mathbf{0}$ has a unique solution), the solution of the optimization problem must deform the mesh $\boldsymbol{x}$ to drive the pair $(\boldsymbol{u}, \boldsymbol{x})$ to a point where the enriched residual is minimized, which we expect to be a mesh that tracks sharp features in the solution. Therefore, we expect this choice of objective function 
to have desirable tracking properties, which will be confirmed by our numerical experiments (Section 6). Finally, this choice of objective function is agnostic to whether we are considering an inviscid or viscous conservation law, which is not the case for many popular physics-based feature indicators than rely, e.g., on the Rankine-Hugoniot conditions.

Our work in [39] considered the objective function in (40), but did not endorse it due to numerical experiments that showed it possessed non-aligned local minima that made it impractical. However, in that work we only considered meshes with a fixed topology, which contributed to these local minima. In the present work, we collapse elements with small volumes after each major optimization iteration, which either eliminates the local minima of (40) or suggests this strategy less sensitive to local minima.

Other choices for the objective function are possible, but are not considered here. The objective function proposed in [39] is the elementwise deviation of the DG solution from its mean, which was shown to have excellent tracking properties. However, it tends to move the mesh in regions of the domain where the solution is smooth and therefore not ideal when the entire mesh is parametrized. The work in [8] uses a physics-based objective function based on the Rankine-Hugoniot conditions at all element faces for inviscid conservation laws, which was shown to work well when combined with a DG-like discretization in a minimum-residual framework.

To not only prevent the degradation of the mesh quality, but actively promote mesh smoothing, we take $\kappa \in \mathbb{R}_{+}$and define $f_{\text {msh }}$ as the deviation of the distortion of the physical mesh from the distortion of the reference mesh

$$
f_{\mathrm{msh}}(\boldsymbol{x})=\frac{1}{2}\left(\boldsymbol{R}_{\mathrm{msh}}(\boldsymbol{x})-\boldsymbol{R}_{\mathrm{msh}}(\boldsymbol{X})\right)^{T}\left(\boldsymbol{R}_{\mathrm{msh}}(\boldsymbol{x})-\boldsymbol{R}_{\mathrm{msh}}(\boldsymbol{X})\right),
$$

where $\boldsymbol{R}_{\mathrm{msh}}: \mathbb{R}^{N_{x}} \rightarrow \mathbb{R}^{\left|\mathcal{E}_{h, q}\right|}$ is the algebraic system corresponding to the elementwise mesh distortion used for high-order mesh generation $[23,14]$

$$
r_{\mathrm{msh}}^{K}\left(\mathcal{G}_{h, q}\right):=\int_{K}\left(\frac{\left\|G_{h, q}\right\|_{F}^{2}}{\left(\operatorname{det} G_{h, q}\right)_{+}^{2 / d}}\right)^{2} d v
$$

and $\boldsymbol{X} \in \mathbb{R}^{N_{x}}$ are the nodal coordinates of the reference mesh. Equation (42) is similar to the distortion measure used in our previous work [39], which was shown to maintain high-quality meshes even when tracking difficult discontinuity surfaces. We define $\boldsymbol{R}_{\mathrm{msh}}$ as the deviation from the distortion of the reference mesh rather than the mesh distortion itself because the Hessian approximation used for our solver performs best when the objective function approaches zero (Section 4.4). Even though the distortion term prevents the combined objective function from converging to zero, if the mesh is high-quality, we expect $\boldsymbol{R}_{\mathrm{msh}}(\boldsymbol{x})$ to be close to its minimum value (component-wise) $\boldsymbol{R}_{\mathrm{msh}}(\boldsymbol{X})$.

To close this section, we define the following vector-valued function

$$
\boldsymbol{F}(\boldsymbol{u}, \boldsymbol{x}):=\left[\begin{array}{c}
\boldsymbol{R}(\boldsymbol{u}, \boldsymbol{x}) \\
\kappa\left(\boldsymbol{R}_{\mathrm{msh}}(\boldsymbol{x})-\boldsymbol{R}_{\mathrm{msh}}(\boldsymbol{X})\right)
\end{array}\right]
$$

and re-write the objective function as

$$
f(\boldsymbol{u}, \boldsymbol{x})=\frac{1}{2}\|\boldsymbol{F}(\boldsymbol{u}, \boldsymbol{x})\|_{2}^{2}=\frac{1}{2} \boldsymbol{R}(\boldsymbol{u}, \boldsymbol{x})^{T} \boldsymbol{R}(\boldsymbol{u}, \boldsymbol{x})+\frac{\kappa^{2}}{2}\left(\boldsymbol{R}_{\mathrm{msh}}(\boldsymbol{x})-\boldsymbol{R}_{\mathrm{msh}}(\boldsymbol{X})\right)^{T}\left(\boldsymbol{R}_{\mathrm{msh}}(\boldsymbol{x})-\boldsymbol{R}_{\mathrm{msh}}(\boldsymbol{X})\right),
$$

to emphasize the objective function is the square two-norm of a residual function. This has implications in terms of available solvers for the optimization problem in (32) as will be discussed in Section 4.4.

\subsection{Boundary constraint enforcement}

In order to maintain a boundary-conforming mesh, the coordinates of all mesh nodes cannot be allowed to move freely; rather, we must add boundary constraints to ensure nodes slide along the domain boundaries. To this end, we write the mesh node coordinates as the result of a mapping $\chi: \mathbb{R}^{N_{\phi}} \rightarrow \mathbb{R}^{N_{x}}$ from the unconstrained degrees of freedom $\phi \in \mathbb{R}^{N_{\phi}}$ that incorporates all boundary constraints

$$
\boldsymbol{x}=\chi(\phi)
$$


where the specific form of the mapping depends on the domain under consideration. This constraint is incorporated into the optimization problem (32) as:

$$
\begin{array}{ll}
\underset{\boldsymbol{u} \in \mathbb{R}^{N_{\boldsymbol{u}}, \boldsymbol{\phi} \in \mathbb{R}^{N_{\boldsymbol{\phi}}}}}{\operatorname{minimize}} & f(\boldsymbol{u}, \chi(\boldsymbol{\phi})) \\
\text { subject to } & \boldsymbol{r}(\boldsymbol{u}, \chi(\boldsymbol{\phi}))=\mathbf{0} .
\end{array}
$$

As discussed in [39], the re-parametrized formulation can also be used to explicitly incorporate mesh smoothing into the mesh deformation. By introducing the following definitions

$$
\tilde{f}(\boldsymbol{u}, \boldsymbol{\phi}):=f(\boldsymbol{u}, \chi(\phi)), \quad \tilde{\boldsymbol{r}}(\boldsymbol{u}, \boldsymbol{\phi}):=\boldsymbol{r}(\boldsymbol{u}, \chi(\phi)), \quad \tilde{\boldsymbol{F}}(\boldsymbol{u}, \boldsymbol{\phi}):=\boldsymbol{F}(\boldsymbol{u}, \chi(\phi)),
$$

the optimization problem with boundary enforcement in (46) becomes

$$
\begin{array}{ll}
\underset{\boldsymbol{u} \in \mathbb{R}^{N_{u}}, \boldsymbol{\phi} \in \mathbb{R}^{N}}{\operatorname{minimize}} & \tilde{f}(\boldsymbol{u}, \boldsymbol{\phi}) \\
\text { subject to } & \tilde{\boldsymbol{r}}(\boldsymbol{u}, \boldsymbol{\phi})=\mathbf{0},
\end{array}
$$

which has the same structure as the original optimization problem without boundary enforcement (32) with the following replacements: $\tilde{f} \leftarrow f, \tilde{\boldsymbol{r}} \leftarrow \boldsymbol{r}$, and $\boldsymbol{\phi} \leftarrow \boldsymbol{x}$.

Most of the problems in this work only require nodes to slide along boundaries aligned with coordinate directions; the nodes on all other boundaries are fixed. Nodes sliding along more general boundaries has been considered in $[11,40]$. In this special case, we partition the mesh node coordinates into the constrained $\boldsymbol{x}_{c} \in \mathbb{R}^{N_{\boldsymbol{x}}-N_{\phi}}$ and unconstrained $\boldsymbol{\phi} \in \mathbb{R}^{N_{\boldsymbol{\phi}}}$ coordinates

$$
\boldsymbol{x}=\left[\begin{array}{c}
\boldsymbol{\phi} \\
\boldsymbol{x}_{c}
\end{array}\right]
$$

which implies the boundary mapping is the padded identity mapping

$$
\boldsymbol{x}=\chi\left(\boldsymbol{\phi} ; \boldsymbol{x}_{c}\right):=\left[\begin{array}{c}
\boldsymbol{\phi} \\
\boldsymbol{x}_{c}
\end{array}\right], \quad \frac{\partial \chi}{\partial \phi}\left(\boldsymbol{\phi} ; \boldsymbol{x}_{c}\right)=\left[\begin{array}{c}
\boldsymbol{I} \\
\mathbf{0}
\end{array}\right] .
$$

\section{Full space, minimum-residual solver for optimization-based discontinuity tracking}

With the formulation of the optimization problem given in (48), this section introduces a robust, iterative solver. For simplicity, we introduce the solver for the optimization problem in (32), i.e., without boundary enforcement; however, due to the mirror structure between (32) and (48), the exact algorithm applies to solve (48) with the following replacements: $\tilde{f} \leftarrow f, \tilde{\boldsymbol{r}} \leftarrow \boldsymbol{r}$, and $\boldsymbol{\phi} \leftarrow \boldsymbol{x}$.

For brevity, we combine the PDE solution $\boldsymbol{u}$ and mesh coordinates $\boldsymbol{x}$ into a single vector

$$
\boldsymbol{z}:=\left[\begin{array}{l}
\boldsymbol{u} \\
\boldsymbol{x}
\end{array}\right] \in \mathbb{R}^{N_{z}},
$$

where $N_{\boldsymbol{z}}=N_{\boldsymbol{u}}+N_{\boldsymbol{x}}$. In the remainder, we will replace $(\boldsymbol{u}, \boldsymbol{x})$ with $\boldsymbol{z}$, and vice versa, as needed. We will also abbreviate the partial derivatives of the objective function and DG residual as

$$
\begin{array}{llll}
\boldsymbol{g}_{\boldsymbol{z}}(\boldsymbol{z})=\frac{\partial f}{\partial \boldsymbol{z}}(\boldsymbol{z})^{T} & \boldsymbol{g}_{\boldsymbol{u}}(\boldsymbol{z})=\frac{\partial f}{\partial \boldsymbol{u}}(\boldsymbol{z})^{T} & \boldsymbol{g}_{\boldsymbol{x}}(\boldsymbol{z})=\frac{\partial f}{\partial \boldsymbol{x}}(\boldsymbol{z})^{T} \\
\boldsymbol{J}_{\boldsymbol{z}}(\boldsymbol{z})=\frac{\partial \boldsymbol{r}}{\partial \boldsymbol{z}}(\boldsymbol{z}), & \boldsymbol{J}_{\boldsymbol{u}}(\boldsymbol{z})=\frac{\partial \boldsymbol{r}}{\partial \boldsymbol{u}}(\boldsymbol{z}), & \boldsymbol{J}_{\boldsymbol{x}}(\boldsymbol{z})=\frac{\partial \boldsymbol{r}}{\partial \boldsymbol{x}}(\boldsymbol{z}) .
\end{array}
$$

\subsection{Sequential quadratic programming solver}

The proposed solver is a sequential quadratic programming (SQP) method [5] that uses a sequence of quadratic programs to solve (32). Let $\boldsymbol{z}_{k}$ denote the current iterate and formulate a quadratic subproblem 
by linearizing the constraint about $\boldsymbol{z}_{k}$ and using a second-order Taylor series centered at $\boldsymbol{z}_{k}$ to approximate the Lagrangian function

$$
\begin{array}{cl}
\underset{\Delta \boldsymbol{z} \in \mathbb{R}^{N \boldsymbol{z}}}{\operatorname{minimize}} & \boldsymbol{g}_{\boldsymbol{z}}\left(\boldsymbol{z}_{k}\right)^{T} \Delta \boldsymbol{z}+\frac{1}{2} \Delta \boldsymbol{z}^{T} \boldsymbol{B}\left(\boldsymbol{z}_{k}\right) \Delta \boldsymbol{z} \\
\text { subject to } & \boldsymbol{r}\left(\boldsymbol{z}_{k}\right)+\boldsymbol{J}_{\boldsymbol{z}}\left(\boldsymbol{z}_{k}\right) \Delta \boldsymbol{z}=\mathbf{0},
\end{array}
$$

where $\boldsymbol{B}(\boldsymbol{z}) \in \mathbb{R}^{N_{z} \times N_{\boldsymbol{z}}}$ a symmetric positive definite approximation of the Hessian of $\mathcal{L}(\boldsymbol{z})$, i.e.,

$$
\boldsymbol{B}(\boldsymbol{z}):=\left[\begin{array}{cc}
\boldsymbol{B}_{u \boldsymbol{u}}(\boldsymbol{z}) & \boldsymbol{B}_{\boldsymbol{u x}}(\boldsymbol{z}) \\
\boldsymbol{B}_{\boldsymbol{u x}}(\boldsymbol{z})^{T} & \boldsymbol{B}_{\boldsymbol{x} \boldsymbol{x}}(\boldsymbol{z})
\end{array}\right]
$$

such that

$$
\begin{aligned}
& \frac{\partial^{2} \mathcal{L}}{\partial \boldsymbol{u} \partial \boldsymbol{u}}(\boldsymbol{z}) \approx \boldsymbol{B}_{\boldsymbol{u} \boldsymbol{u}}(\boldsymbol{z}) \in \mathbb{R}^{N_{\boldsymbol{u}} \times N_{\boldsymbol{u}}} \\
& \frac{\partial^{2} \mathcal{L}}{\partial \boldsymbol{u} \partial \boldsymbol{x}}(\boldsymbol{z}) \approx \boldsymbol{B}_{\boldsymbol{u} \boldsymbol{x}}(\boldsymbol{z}) \in \mathbb{R}^{N_{\boldsymbol{u}} \times N_{\boldsymbol{x}}} \\
& \frac{\partial^{2} \mathcal{L}}{\partial \boldsymbol{x} \partial \boldsymbol{x}}(\boldsymbol{z}) \approx \boldsymbol{B}_{\boldsymbol{x} \boldsymbol{x}}(\boldsymbol{z}) \in \mathbb{R}^{N_{\boldsymbol{x}} \times N_{\boldsymbol{x}}} .
\end{aligned}
$$

and the dependence on the Lagrange multipliers has been dropped. Once the quadratic subproblem is solved to obtain the current search direction $\Delta \boldsymbol{z}_{k+1} \in \mathbb{R}^{N_{z}}$, the current iterate is updated according to

$$
\boldsymbol{z}_{k+1}=\boldsymbol{z}_{k}+\alpha_{k+1} \Delta \boldsymbol{z}_{k+1}
$$

to yield a sequence of iterates $\left\{\boldsymbol{z}_{k}\right\}$ where $\alpha_{k+1} \in(0,1]$ is a step length parameter (Section 4.3). If $\boldsymbol{B}$ is the true Hessian and $\alpha=1$, this method is equivalent to Newton's method applied to the first-order optimality conditions of (32) [5] and therefore will converge quadratically provided the initial guess is sufficiently close to a local minima.

\subsection{Linear subproblem}

The first-order optimality condition of the quadratic program (53) leads to the following linear system of equations

$$
\left[\begin{array}{ccc}
\boldsymbol{B}_{\boldsymbol{u u}}\left(\boldsymbol{z}_{k}\right) & \boldsymbol{B}_{\boldsymbol{u x}}\left(\boldsymbol{z}_{k}\right) & \boldsymbol{J}_{\boldsymbol{u}}\left(\boldsymbol{z}_{k}\right)^{T} \\
\boldsymbol{B}_{\boldsymbol{u x}}\left(\boldsymbol{z}_{k}\right)^{T} & \boldsymbol{B}_{\boldsymbol{x x}}\left(\boldsymbol{z}_{k}\right) & \boldsymbol{J}_{\boldsymbol{x}}\left(\boldsymbol{z}_{k}\right)^{T} \\
\boldsymbol{J}_{\boldsymbol{u}}\left(\boldsymbol{z}_{k}\right) & \boldsymbol{J}_{\boldsymbol{x}}\left(\boldsymbol{z}_{k}\right) & \mathbf{0}
\end{array}\right]\left[\begin{array}{c}
\Delta \boldsymbol{u}_{k+1} \\
\Delta \boldsymbol{x}_{k+1} \\
\boldsymbol{\eta}_{k+1}
\end{array}\right]=-\left[\begin{array}{c}
\boldsymbol{g}_{\boldsymbol{u}}\left(\boldsymbol{z}_{k}\right) \\
\boldsymbol{g}_{\boldsymbol{x}}\left(\boldsymbol{z}_{k}\right) \\
\boldsymbol{r}\left(\boldsymbol{z}_{k}\right)
\end{array}\right],
$$

where $\boldsymbol{\eta}_{k+1} \in \mathbb{R}^{N_{u}}$ are the Lagrange multipliers associated with the linearized constraint and the step $\Delta \boldsymbol{z}_{k+1}$ is decomposed as a step in the PDE state $\Delta \boldsymbol{u}_{k+1}$ and the nodal mesh coordinates $\Delta \boldsymbol{x}_{k+1}$

$$
\Delta \boldsymbol{z}_{k+1}=\left[\begin{array}{c}
\Delta \boldsymbol{u}_{k+1} \\
\Delta \boldsymbol{x}_{k+1}
\end{array}\right]
$$

The state update $\boldsymbol{\Delta} \boldsymbol{u}$ and Lagrange multipliers $\boldsymbol{\eta}$ can be eliminated from the linear system to obtain an explicit expression for the state and mesh updates

$$
\Delta \boldsymbol{x}_{k+1}=-\boldsymbol{A}\left(\boldsymbol{z}_{k}\right)^{-1} \boldsymbol{b}\left(\boldsymbol{z}_{k}\right), \quad \Delta \boldsymbol{u}_{k+1}=\Delta \boldsymbol{u}_{0}\left(\boldsymbol{z}_{k}\right)+\boldsymbol{C}\left(\boldsymbol{z}_{k}\right) \Delta \boldsymbol{x},
$$

where

$$
\begin{aligned}
\Delta \boldsymbol{u}_{0}(\boldsymbol{z}) & =-\boldsymbol{J}_{\boldsymbol{u}}(\boldsymbol{z})^{-1} \boldsymbol{r}(\boldsymbol{z}) \\
\boldsymbol{C}(\boldsymbol{z}) & =-\boldsymbol{J}_{\boldsymbol{u}}(\boldsymbol{z})^{-1} \boldsymbol{J}_{\boldsymbol{x}}(\boldsymbol{z}) \\
\boldsymbol{A}(\boldsymbol{z}) & =\boldsymbol{B}_{\boldsymbol{x} \boldsymbol{x}}(\boldsymbol{z})+2 \boldsymbol{C}(\boldsymbol{z})^{T} \boldsymbol{B}_{\boldsymbol{u} \boldsymbol{x}}(\boldsymbol{z})+\boldsymbol{C}(\boldsymbol{z})^{T} \boldsymbol{B}_{\boldsymbol{u} \boldsymbol{u}}(\boldsymbol{z}) \boldsymbol{C}(\boldsymbol{z}) \\
\boldsymbol{b}(\boldsymbol{z}) & =\boldsymbol{g}_{\boldsymbol{x}}(\boldsymbol{z})+\boldsymbol{C}(\boldsymbol{z})^{T} \boldsymbol{g}_{\boldsymbol{u}}(\boldsymbol{z})+\left(\boldsymbol{B}_{\boldsymbol{u} \boldsymbol{x}}(\boldsymbol{z})^{T}+\boldsymbol{C}(\boldsymbol{z})^{T} \boldsymbol{B}_{\boldsymbol{u} \boldsymbol{u}}(\boldsymbol{z})\right) \Delta \boldsymbol{u}_{0} .
\end{aligned}
$$

Notice $\Delta \boldsymbol{u}_{0}(\boldsymbol{z})$ is the Newton step for the nonlinear system $\boldsymbol{r}(\boldsymbol{u}, \boldsymbol{x})=\mathbf{0}$ if $\boldsymbol{x}$ is fixed and $\boldsymbol{C}(\boldsymbol{z})$ is the sensitivity of the solution $\boldsymbol{u}$ with respect to the mesh nodes $\boldsymbol{x}$.

The linear systems in (57) and (59) are two mathematically equivalent options to compute the solution to the SQP subproblem that place different requirements on the linear solver. The system in (57) is large 
(size: $2 N_{\boldsymbol{u}}+N_{\boldsymbol{x}}$ ) since it simultaneously computes the steps $\Delta \boldsymbol{u}, \Delta \boldsymbol{x}$ and Lagrange multipliers $\boldsymbol{\eta}$, but each (block) entry is relatively easy to form. On the other hand, the system in (59) is smaller (size: $N_{\boldsymbol{x}}$ ), but formation of $\boldsymbol{A}\left(\boldsymbol{z}_{k}\right)$ requires computation of $\boldsymbol{C}\left(\boldsymbol{z}_{k}\right)$, which is impractical if a direct solver is not available. In this work, we use a direct solver to solve (57); however, this approach is not practical for large-scale problems. In future work, we will develop iterative solvers and preconditioners for the full system in (57).

\subsection{Line search globalization}

To ensure the sequence $\left\{\boldsymbol{z}_{k}\right\}$ converges to a first-order critical point of (32) from an arbitrary initial guess, the SQP algorithm must be globalized with e.g., a trust region strategy or line search. We choose to globalize the SQP method with a line search that computes the step length such that it minimizes a merit function that combines the objective function and a measure of constraint violation into a scalar function. In this work, we use the $\ell_{1}$ penalty function $\varphi_{k}: \mathbb{R} \rightarrow \mathbb{R}$

$$
\varphi_{k}(\alpha):=f\left(\boldsymbol{z}_{k}+\alpha \Delta \boldsymbol{z}_{k}\right)+\mu\left\|\boldsymbol{r}\left(\boldsymbol{z}_{k}+\alpha \Delta \boldsymbol{z}_{k+1}\right)\right\|_{1}
$$

where $\mu>0$ is the penalty parameter. This is an exact merit function in the sense that there exists a positive scalar $\hat{\mu}$ such that for any $\mu>\hat{\mu}$, any local solution of (32) is a local minimizer of (61) [27]. Furthermore, $\hat{\mu}=\left\|\boldsymbol{\lambda}^{\star}\right\|_{\infty}$, where $\boldsymbol{\lambda}^{\star}$ are the Lagrange multipliers associated with the optimal solution $\left(\boldsymbol{u}^{\star}, \boldsymbol{x}^{\star}\right)$ [27]. Therefore, in the present setting we take $\mu=2\left\|\hat{\boldsymbol{\lambda}}\left(\boldsymbol{z}_{k}\right)\right\|_{\infty}$, where $\hat{\boldsymbol{\lambda}}$ is defined in (36).

It is well-known that it is not necessary to find the exact minimizer of the merit function to obtain a convergent algorithm. Instead, we search for $\alpha_{k+1} \in(0,1]$ that satisfies sufficient decrease

$$
\varphi_{k}\left(\alpha_{k+1}\right) \leqslant \varphi_{k}(0)+c \alpha_{k+1} \varphi_{k}^{\prime}(0)
$$

where $c \in(0,1)$. We use a backtracking strategy [27] to determine $\alpha_{k+1}$ : define $\alpha_{k+1}=\tau^{n-1}$ for $\tau \in(0,1)$ and let $n \in \mathbb{N}$ be the smallest number such that sufficient decrease (62) holds. In this work we make standard choices for these parameters [27]: $c=10^{-4}$ and $\tau=0.5$.

\subsection{Levenberg-Marquardt Hessian approximation}

The true Hessian of the Lagrangian in (33) is

$$
\boldsymbol{H}(\boldsymbol{z})=\frac{\partial \boldsymbol{F}}{\partial \boldsymbol{z}}(\boldsymbol{z})^{T} \frac{\partial \boldsymbol{F}}{\partial \boldsymbol{z}}(\boldsymbol{z})+\boldsymbol{F}_{i}(\boldsymbol{z}) \frac{\partial^{2} \boldsymbol{F}_{i}}{\partial \boldsymbol{z} \partial \boldsymbol{z}}(\boldsymbol{z})-\boldsymbol{\lambda}_{i} \frac{\partial^{2} \boldsymbol{r}_{i}}{\partial \boldsymbol{z} \partial \boldsymbol{z}}(\boldsymbol{z})
$$

where summation is implied over the repeated index. The second and third term involves the Hessian of the DG residual, which is a complicated third-order tensor that is rarely available in computational mechanics codes. Therefore, we use the Gauss-Newton assumption and approximate the Hessian as

$$
\boldsymbol{H}(\boldsymbol{z}) \approx \frac{\partial \boldsymbol{F}}{\partial \boldsymbol{z}}(\boldsymbol{z})^{T} \frac{\partial \boldsymbol{F}}{\partial \boldsymbol{z}}(\boldsymbol{z})
$$

which is justified if the combined enriched residual and mesh distortion $\boldsymbol{F}(\boldsymbol{z})$ is small. While this approximation is convenient, it could also lead to singular or ill-conditioned Hessian approximations, which would in turn lead to poor search directions. Therefore, we use the Levenberg-Marquardt approach that adds a scaled multiple of a symmetric positive definite matrix to regularize the system. Based on the observations in [8] that Levenberg-Marquardt regularization improves the mesh motion and is not needed for the state, we only regularize the mesh components of the Hessian, i.e.,

$$
\boldsymbol{B}_{\boldsymbol{u} \boldsymbol{u}}(\boldsymbol{z})=\frac{\partial \boldsymbol{F}}{\partial \boldsymbol{u}}(\boldsymbol{z})^{T} \frac{\partial \boldsymbol{F}}{\partial \boldsymbol{u}}(\boldsymbol{z}), \quad \boldsymbol{B}_{\boldsymbol{u x}}(\boldsymbol{z})=\frac{\partial \boldsymbol{F}}{\partial \boldsymbol{u}}(\boldsymbol{z})^{T} \frac{\partial \boldsymbol{F}}{\partial \boldsymbol{x}}(\boldsymbol{z}), \quad \boldsymbol{B}_{\boldsymbol{x} \boldsymbol{x}}(\boldsymbol{z})=\frac{\partial \boldsymbol{F}}{\partial \boldsymbol{x}}(\boldsymbol{z})^{T} \frac{\partial \boldsymbol{F}}{\partial \boldsymbol{x}}(\boldsymbol{z})+\gamma \boldsymbol{D},
$$

where $\gamma \in \mathbb{R}_{+}$and $\boldsymbol{D} \in \mathbb{R}^{N_{x} \times N_{x}}$ is a symmetric positive definite (SPD) matrix (Section 4.5).

\subsection{Choice of regularization matrix}

The classic Levenberg-Marquardt algorithm uses the identity matrix as the regularization matrix $\boldsymbol{D}=$ $\boldsymbol{I}_{N_{\boldsymbol{x}}}$ to guard against poor search directions that could result if the Jacobian of $\boldsymbol{F}$ with respect to $\boldsymbol{x}$ is rank 
deficient or ill-conditioned. However, the same result can be achieved for any SPD matrix $\boldsymbol{D}$. We consider a regularization matrix that is known to possess smoothing properties: the stiffness matrix of a linear elliptic PDE. To this end, define a vector-valued function $v: \Omega_{0} \rightarrow \mathbb{R}^{d}$ in which each component satisfies the elliptic PDE with homogeneous Neumann boundary conditions

$$
\nabla_{X} \cdot\left(k \nabla_{X} v_{i}\right)=0 \quad \text { in } \Omega_{0}, \quad \nabla_{X} v_{i} \cdot N=0 \quad \text { on } \partial \Omega_{0}
$$

for $i=1, \ldots, d$ and the coefficient $k: \Omega_{0} \rightarrow \mathbb{R}_{+}$is piecewise constant over each element in $\mathcal{E}_{h, q}$. Numerical experimentation on problems where elements in the reference mesh significantly vary in size indicate that the natural scaling of the stiffness matrix $(k=1)$ is not sufficient to provide search directions that are scaled according to the mesh resolution. Instead, we take the piecewise constant coefficient as

$$
k(x)=\omega_{K}, \quad x \in K
$$

for each $K \in \mathcal{E}_{h, q}$, where $\omega_{K} \in \mathbb{R}_{+}$is inversely proportional to the size of the element

$$
\omega_{K}:=\frac{\min _{K^{\prime} \in \mathcal{E}_{h, q}}\left|K^{\prime}\right|}{|K|} .
$$

The regularization matrix is the assembled stiffness matrix associated with the elliptic system in (66)(67) over the global finite element space $\mathcal{W}_{h, q}$. This is similar to the regularization matrix used in [11] without volume-based scaling of the elliptic coefficient, which is important for problems with reference domain elements of varying size (Section 6.3.2). Boundary constraints are incorporated into the elliptic regularization matrix as homogeneous Dirichlet boundary conditions.

\subsection{Adaptive regularization parameter}

The ideal value for the regularization parameter $\gamma$ in (65) is difficult to know a priori. Large values of $\gamma$ produce highly regularized search directions $\Delta \boldsymbol{x}$, e.g., the search direction becomes the solution of the elliptic PDE with the steepest descent direction as the right-hand side; however, in this case, the Hessian approximation is poor. Alternatively, small values of $\gamma$, particularly in early iterations, can leads to bad search directions due to ill-conditioning of the Hessian approximation. Following the work in [8], we introduce an adaptive algorithm to minimize the importance of choosing the appropriate value for $\gamma$ a priori. Our algorithm is based on the heuristic that $\gamma$ should control the size of the mesh deformation at a given iteration. Therefore, if a step is too large, we increase the value of $\gamma$ for the next iteration and vice versa. Let $\gamma_{k}>0$ be the value of the regularization parameter at iteration $k$ and define constants $\kappa_{1}, \kappa_{2}>0, \sigma \in(0,1), \gamma_{\min }>0$ then

$$
\gamma_{k+1}=\max \left\{\bar{\gamma}_{k+1}, \gamma_{\text {min }}\right\}, \quad \bar{\gamma}_{k+1}= \begin{cases}\sigma^{-1} \gamma_{k} & \text { if }\left\|\Delta \boldsymbol{x}_{k}\right\|<\kappa_{1} \\ \sigma \gamma_{k} & \text { if }\left\|\Delta \boldsymbol{x}_{k}\right\|>\kappa_{2} \\ \gamma_{k} & \text { otherwise. }\end{cases}
$$

For domains with dimensions $\mathcal{O}(1)$, we take $\kappa_{1}=10^{-2}$ and $\kappa_{2}=10^{-1}$. In this work, we choose a conservative value $\sigma=0.5$ to prevent $\gamma$ from changing significantly between iterations. The appropriate value of $\gamma_{\min }$ is problem-dependent and will be addressed in Section 6 .

\subsection{Termination criteria}

The termination criteria for the SQP method comes from the first-order optimality criteria discussed in Section 3.1 (37). That is, given tolerances $\epsilon_{1}, \epsilon_{2}>0, \boldsymbol{z}_{k}$ is a considered a numerical solution of (32) if

$$
\left\|\boldsymbol{c}\left(\boldsymbol{z}_{k}\right)\right\|<\epsilon_{1}, \quad\left\|\boldsymbol{r}\left(\boldsymbol{z}_{k}\right)\right\|<\epsilon_{2} .
$$

We have empirically observed that feasibility can be driven to near machine tolerance (take $\epsilon_{2}=10^{-10}$ ); however, the optimality condition is a more difficult condition (take $\epsilon_{1}=10^{-5}$ ). For difficult problems, it may require a large number of iterations, so we safeguard the algorithm with a maximum number of iterations 
$\left(N_{\max }\right)$, i.e., iterations terminate when either the convergence criteria (70) are met or a $N_{\max }$ iterations have been completed. If termination is based on $N_{\max }$, it is likely the DG constraint is not satisfied, i.e.,

$$
\boldsymbol{r}\left(\boldsymbol{z}_{N_{\max }}\right)>\epsilon_{2}
$$

which could lead to a non-conservative scheme. In this case, we solve the DG equations on the fixed mesh with nodal coordinates $\boldsymbol{x}_{N_{\max }}$ using Newton's method.

\section{Practical considerations}

\subsection{Solution and mesh initialization}

The discontinuity-tracking optimization problem in (32) is non-convex and therefore the initial guess for the SQP solver is critical to obtain a good solution. In the present context, this means we must provide a reasonable initial guess for the mesh coordinates $\boldsymbol{x}_{0}$ and DG solution $\boldsymbol{u}_{0}$.

\subsubsection{Special case: straight-sided mesh $(q=1)$}

First consider the case of a straight-sided mesh $(q=1)$ combined with any finite element space for the solution $(p \geqslant 0)$. The mesh is always initialized from the reference mesh which comes from mesh generation agnostic to the discontinuity because we usually do not have an estimate of the discontinuity surface. In special cases where an estimate of the discontinuity surface is available, this could be used to drive generation of the reference mesh. The DG solution is initialized from the DG $(p=0)$ solution on the reference mesh; $p=0$ is used because nonlinear instabilities resulting from oscillations about discontinuities cannot arise with a piecewise constant solution field. For nonlinear problems the $p=0$ solution is obtained using pseudo-transient continuation [21] with adaptive time steps, initialized from uniform flow.

\subsubsection{General case: high-order meshes $(q>1)$}

To avoid local minima in the optimization problem (32) that arise when high-order meshes are used, we usually initialize the tracking problem for $p \geqslant 0, q>1$ from the solution of the tracking problem for $p^{\prime} \leqslant p$, $q^{\prime}=1$. That is, we solve the the tracking problem using a solution space with polynomial degree $p^{\prime} \leqslant p$ and mesh deformation with polynomial degree $q^{\prime}=1$ and initialize the desired tracking problem (solution space of degree $p$ and mesh of degree $q>1$ ) from the resulting DG solution and mesh. This strategy comes from our observations that $q=1$ tracking is quite robust and convergence of the $q>1$ solution from a straight-sided tracking mesh is rapid. For difficult problems, it may be helpful to use continuation on the polynomial degree for the solution as well, i.e., take $p^{\prime}<p$.

\subsection{Edge collapses and solution transfer}

As we will show in our numerical experiments in Section 6, we typically start with an initial mesh that is far from alignment with the shock and iterate using an SQP solver which attempts to move nodes onto the shock. Since the deformation of the initial mesh can be quite large, this can result in severely ill-conditioned elements that drastically degrade the quality of our solution. To address this, we follow the approach in [8] and collapse elements once they become problematic. In particular, after each SQP iteration, we compute the volume of each element $K$ in the physical domain, $v^{K}$, and compare it to the volume of the corresponding element in the reference domain, $v_{0}^{K}$, where

$$
v_{0}^{K}=\int_{K} d V, \quad v^{K}=\int_{\mathcal{G}_{h, q}(K)} d V
$$

If the volume of an element has decreased more than a certain factor, i.e., $v^{K}<\epsilon v_{0}^{K}$ (where $\epsilon=0.2$ in this work), the element is tagged for removal. In principle, $\kappa$ in the objective function (39) could be chosen large enough to prevent these ill-conditioned elements from ever appearing. However, such a $\kappa$ would weight $f_{\mathrm{msh}}$ too heavily over $f_{\text {err }}$ and interfere with the tracking capabilities of the method. Therefore, this weighting term $\kappa$ is only chosen large enough to prevent unacceptably bad elements (such as tangled ones) which would cause the mesh distortion term $f_{\text {msh }}$ to blow up dramatically. As long as we avoid this situation, we can handle the remainder of the ill-conditioned elements through collapses. 
Each element in the physical domain $\boldsymbol{x}$ tagged for removal is eliminated by an edge collapse [25]. For a given tagged element, we choose to collapse the shortest edge into the longest edge (Figure 3). This is based off the principle that the shortest edge is likely to be transverse to the shock and the longest edge is likely to be aligned with the shock. A special case to note is when tagged elements have nodes or edges in common. Additional care must be taken to choose an edge to collapse that is consistent with all affected elements. For elements on the boundary, this logic is slightly modified to ensure that an edge collapse does not move nodes off the boundary. Note that an edge collapse must also be applied to the corresponding element in the reference domain $\boldsymbol{X}$ to ensure the physical and reference domains always have the same topology.

The solution $\boldsymbol{u}$ is transferred to the new mesh by removing the entries corresponding to the degrees of freedom in the collapsed elements. To update the data structures for both the mesh and the solution, we simply need to delete the entries in the element connectivity matrix and solution vector corresponding to the collapsed elements and renumber based off the new node numbering. Because of our choice of a DG discretization, this update is particularly easy to do, and furthermore, removes the need to modify the degrees of freedom in the neighboring elements. In a CG framework, the degrees of freedom in the neighboring elements would need to be modified in order to guarantee continuity on the new mesh.

While these edge collapses do modify the objective function and formally result in a new optimization problem, in practice this does not lead to issues in the convergence of the solver. We observe that these collapses mostly occur in the initial iterations of the SQP solver when the mesh is far from convergence and each iteration results in a large update to the mesh. At some point, the line search described in Section 4.3 will reject steps that would cause large deformations in the mesh, hence precluding the need for further collapses and ensuring that they do not occur indefinitely.
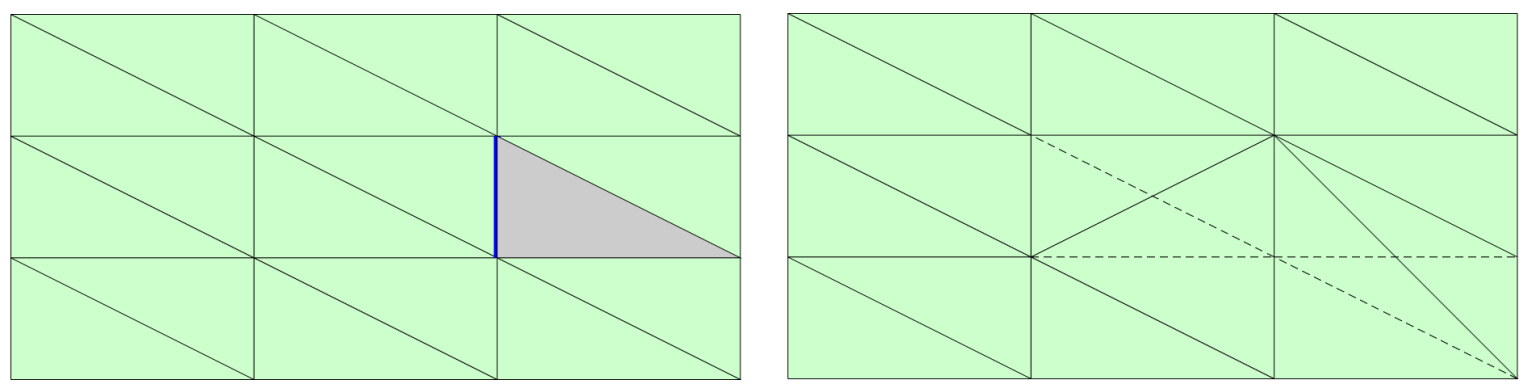

Figure 3: Demonstration of edge collapse algorithm: the element identified in the original mesh (left) is collapsed along the highlighted edge to produce the new mesh (right) with the original elements shown in dashed lines for reference.

\section{Numerical experiments}

In this section we introduce three inviscid conservation laws and demonstrate the tracking framework on six problems with discontinuous solutions of varying difficulty. We also provide a detailed study of the various algorithmic parameters introduced in Section 2-3, in particular, the choice of numerical flux $(\mathcal{H})$ and mesh distortion parameter $(\kappa)$.

\subsection{Linear advection}

The first conservation law we consider is steady linear advection of a scalar quantity $U: \Omega \rightarrow \mathbb{R}$ through a domain $\Omega \subset \mathbb{R}^{d}$

$$
\nabla \cdot(\beta U)=0 \quad \text { in } \Omega, \quad U=U_{\infty} \quad \text { on } \Gamma_{i}
$$

where $\beta: \Omega \rightarrow \mathbb{R}^{d}$ is the local flow direction, $\Gamma_{i}:=\{x \in \partial \Omega \mid \beta \cdot n(x)<0\}$ is the inflow boundary, $\partial \Omega: \mathbb{R}^{d} \rightarrow$ is the unit outward normal to the boundary, and $U_{\infty}: \Gamma_{i} \rightarrow \mathbb{R}$ is the inflow boundary condition. We consider the pure upwind numerical flux $\mathcal{H}_{\text {up }}$ introduced in (25) because it satisfies conditions (i)-(ii) (Section 2.3) for a stable DG discretization and condition (iii) to be suitable for tracking, as well as its smoothed version $\mathcal{H}_{\mathrm{up}}^{a}$ to recover smoothness with respect to variations of the mesh nodes (iv). In this section we take the smoothness parameter to be $a=10$ to trade-off between smoothness and appropriate upwinding. The boundary condition is enforced via the pure upwind numerical flux evaluated at the interior state $U^{+}$and the boundary state $U^{\partial}=U_{\infty}$. 


\subsubsection{Straight shock, piecewise constant solution}

First we consider a two-dimensional $(d=2)$ domain $\Omega:=(-1,1) \times(0,1)$ with constant advection field and piecewise constant boundary condition

$$
\beta(x)=\left[\begin{array}{c}
-1.25 \\
1
\end{array}\right], \quad U_{\infty}(x)=H(x) .
$$

This leads to a linear discontinuity surface $\Gamma_{s} \subset \Omega$

$$
\Gamma_{s}:=\{(-1.25 s, s) \mid s \in(0,0.8)\}
$$

and piecewise constant solution field

$$
U(x)=H\left(x_{1}+1.25 x_{2}\right) .
$$

To ensure the Heaviside function on the bottom (inflow) boundary is accurately represented and integrated in the weak form, we require our computational mesh to have an element face that intersects $(0,0)$ and do not allow the corresponding node to move throughout iterations using the boundary mapping described in Section 3.3.

Given the piecewise constant solution and linear shock surface, the solution lies in a $p=0$ polynomial basis on a piecewise linear mesh $q=1$, provided the mesh tracks the discontinuity. To demonstrate the performance of the tracking method, we use this minimal basis with $p=0, q=1$. The reference mesh is taken to be a uniform triangular mesh of the domain with 36 elements. The mesh and solution are initialized according to Section 5.1. The SQP solver is used with $\lambda$ chosen adaptively (Section 4.6). The various DG/tracking parameters are set as follows: $\kappa=0$ (no mesh smoothing), pure upwind numerical flux $(a=\infty), \gamma_{0}=10^{-2}$ and $\gamma_{\min }=10^{-8}$ (regularization parameter adaptivity), and $\epsilon_{1}=10^{-10}$, and $\epsilon_{2}=10^{-12}$ (termination criteria). After only 10 iterations, the mesh perfectly tracks the discontinuity (Figure 4) and the DG solution closely matches the exact solution; the $L^{1}$ error of the solution is $3.84 \times 10^{-11}$. For this problem where the finite element subspace contains the exact solution of the problem, the tracking method exhibits Newton-like convergence (Figure 5). Furthermore, the tracking solver is robust with regard to the various algorithmic parameters introduced, i.e., fast convergence to the exact solution was obtained without mesh smoothing, with the upwind numerical flux (not smooth with respect to domain deformation), and nearly independent of the choice of regularization adaptivity $\left(\gamma_{0}, \gamma_{\min }\right)$. The choice of these parameters becomes significant for curved discontinuities and nontrivial flows as we will demonstrate in subsequent sections.

\subsubsection{Curved shock, piecewise constant solution}

Next we consider linear advection through a two-dimensional $(d=2)$ domain $\Omega:=(-1,1) \times(0,1)$ with a spatially varying advection field and piecewise constant boundary condition

$$
\beta(x)=\left[\begin{array}{c}
-\sin \left(\pi x_{2}\right) \\
1
\end{array}\right], \quad U_{\infty}(x)=H(x) .
$$

This leads to a trigonometric discontinuity surface $\Gamma_{s} \subset \Omega$

$$
\Gamma_{s}:=\left\{\left(\frac{\cos (\pi s)-1}{\pi}, s\right) \mid s \in(0,1)\right\}
$$

and piecewise constant solution field

$$
U(x)=H\left(\pi x_{1}-\cos \left(\pi x_{2}\right)+1\right) .
$$

Unlike the previous problem, this solution cannot be represented exactly using polynomial basis functions since the discontinuity surface is non-polynomial (trigonometric). We use a piecewise constant solution basis $(p=0)$ and piecewise linear, quadratic, and cubic basis for the mesh $(q=1,2,3)$. The reference mesh is taken to be a uniform triangular mesh of the domain with 64 elements. The mesh and solution are initialized according to Section 5.1; however, for this problem we do not use continuation in the polynomial degree. The SQP solver is used with $\lambda$ chosen adaptively (Section 4.6). The various DG/tracking parameters are set 
Iteration 0

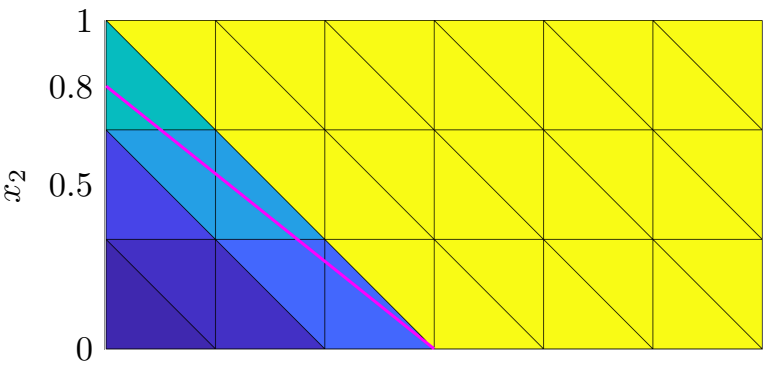

Iteration 2

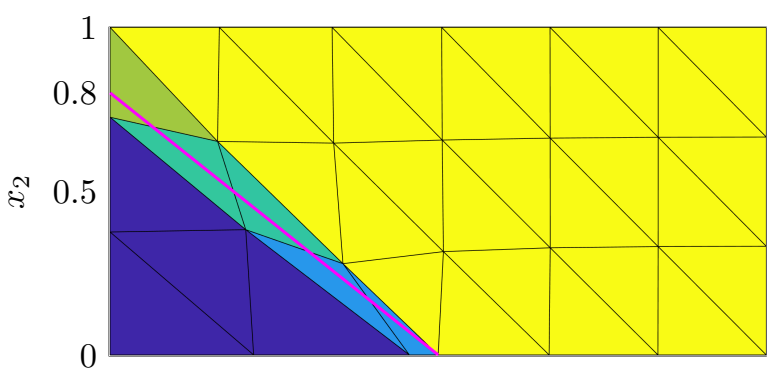

Iteration 4

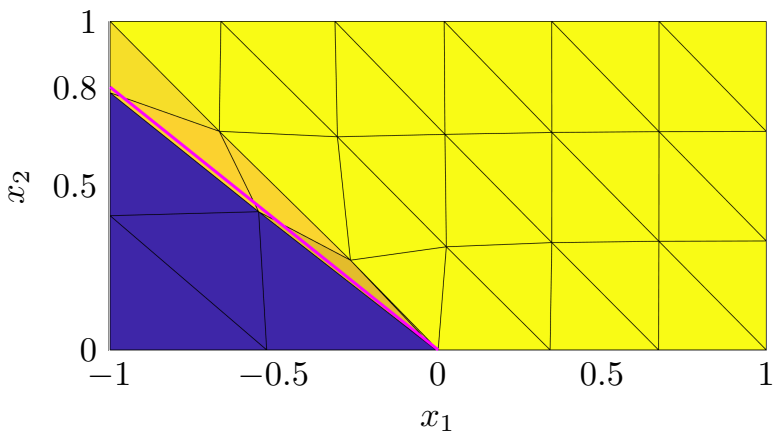

Iteration 1

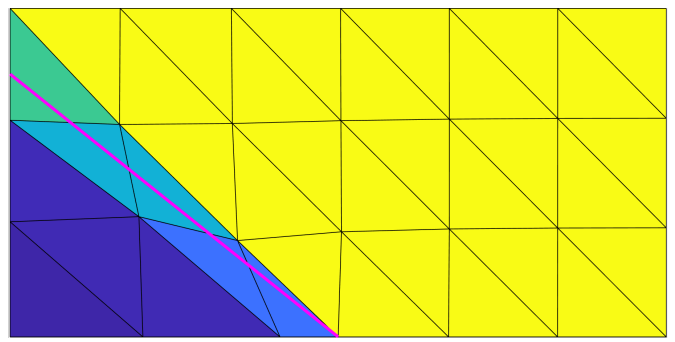

Iteration 3

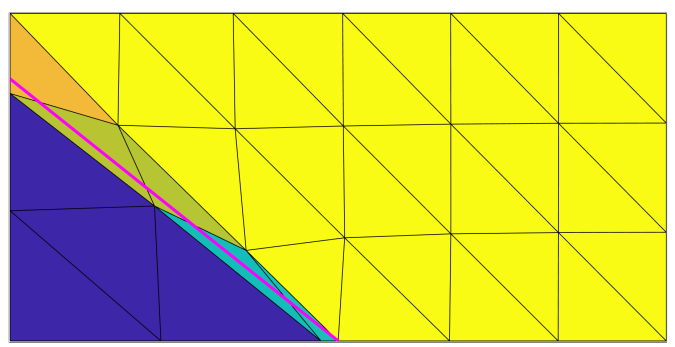

Iteration 10

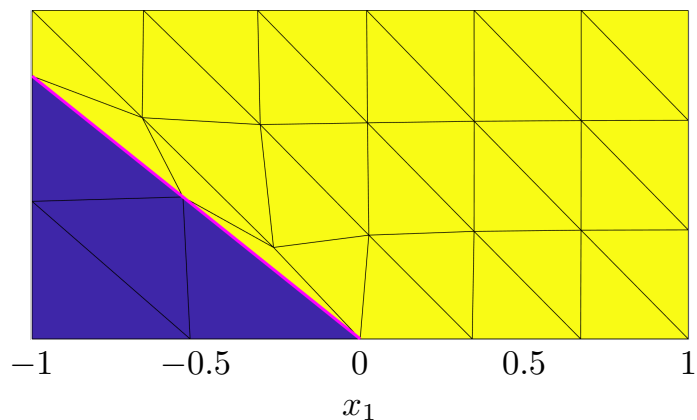

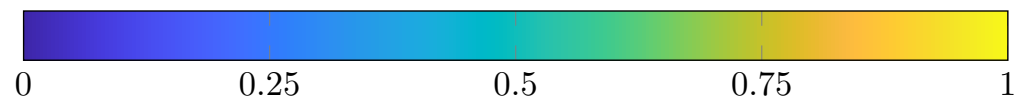

Figure 4: Solution of advection equation with a straight shock (constant advection field) using the tracking method at various iterations throughout the solution procedure using $p=0$ basis for the solution and $q=1$ basis for the mesh. The magenta line highlights the discontinuity surface of the exact solution. The method converges to the exact solution, which lies in the chosen finite element subspace, to near machine precision in only 10 iterations. 

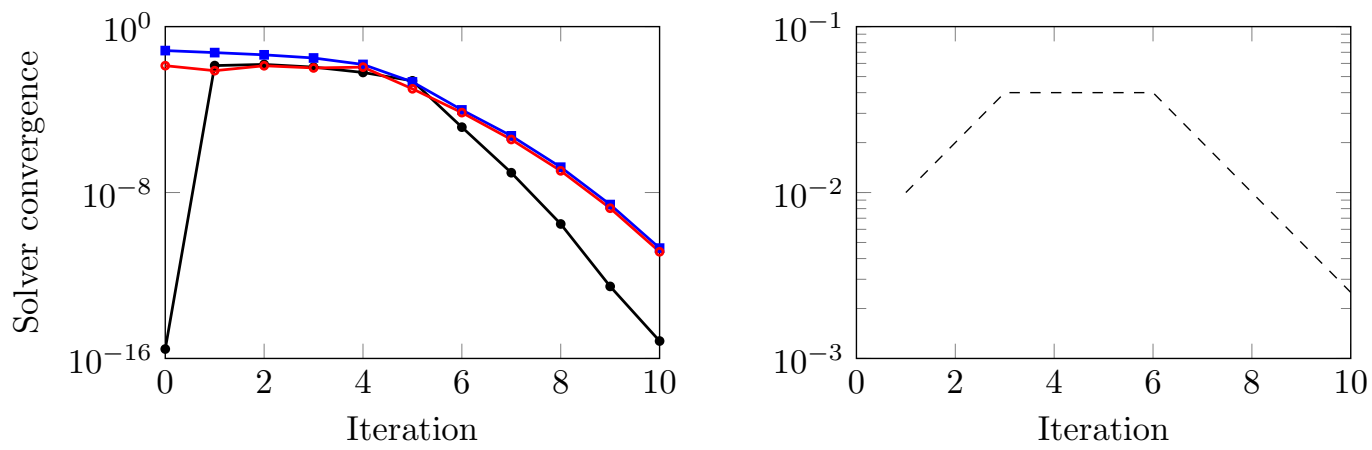

Figure 5: Convergence of the DG residual $\|\boldsymbol{r}(\boldsymbol{u}, \boldsymbol{x})\|(\longrightarrow-)$, enriched DG residual $\|\boldsymbol{R}(\boldsymbol{u}, \boldsymbol{x})\|(\rightarrow-)$, optimality condition $\|\boldsymbol{c}(\boldsymbol{u}, \boldsymbol{x})\|(--)$, and control of the regularization parameter $\left(--_{-}\right)$for the tracking method applied to the advection equation with a straight shock (constant advection field). For this simple problem, Newton-like convergence is achieved.

as follows: $\kappa=0$ (mesh smoothing), smoothed upwind numerical flux $(a=10), \gamma_{0}=10^{-2}$ and $\gamma_{\min }=10^{-3}$ (regularization parameter adaptivity), and $N_{\max }=80, \epsilon_{1}=10^{-10}, \epsilon_{2}=10^{-12}$ (termination criteria). For all polynomial degrees considered, the mesh tracks the discontinuity as accurately as possible given the resolution in the finite element space (Figure 6). The $q=1$ solution is under-resolved since the combination of the coarse mesh and straight-sided elements is not sufficient to resolve the discontinuity structure and as a result the solution exhibits over- and under-shoot; the $L^{1}$ error associated with this solution is $5.79 \times 10^{-2}$. However, the $q=2$ and $q=3$ solutions are extremely accurate even on the coarse mesh due to the high-order elements that curve to conform to the discontinuity structure; the $L^{1}$ error associated with each solution is $1.15 \times 10^{-3}$ and $5.50 \times 10^{-4}$, respectively.

For all polynomial degrees considered, the solver is able to converge the KKT system to relatively tight tolerances $\left(\|\boldsymbol{r}(\boldsymbol{u}, \boldsymbol{x})\|<10^{-10}\right.$ and $\left.\|\boldsymbol{c}(\boldsymbol{u}, \boldsymbol{x})\|<10^{-7}\right)$. As the polynomial degree increases, the enriched residual and mesh distortion converge to increasingly small values and the overall convergence of the solver becomes cleaner (Figure 7). This comes from the improved Hessian approximation, which comes from the mesh distortion and enriched residual converging to smaller values and justifies dropping of the second term in (64). The regularization parameter is adapted to control the size of $\Delta \boldsymbol{x}$ produced from the linear solve and the line search ensures sufficient progress is made with respect to the $\ell_{1}$ merit function (Figure 7 ). For this problem, a non-unity step size is only required once the regularization parameter is small, which is required near convergence to have a decent approximation to the Hessian.

For this problem, the mesh smoothing parameter $(\kappa)$ and minimum value for the regularization parameter $\left(\gamma_{\min }\right)$ were important for the $q=1$ tracking problem; for $\kappa<10^{-2}$ or $\gamma_{\min }<10^{-3}$, the tracking algorithm would continually collapse elements near the discontinuity as it pushed nodes to this region to improve the faceted approximation of the trigonometric discontinuity surface. For $q>1$, the smoothing parameter played no role as the mesh is well-conditioned even with $\kappa=0$ and $\gamma_{\min }$ can be taken much smaller, e.g., $\gamma_{\min }=10^{-8}$, without adversely affecting the performance of the solver or the final solution. The numerical flux plays a more significant role with regard to the behavior of the SQP solver, even for higher order elements. For the non-smooth (with respect to domain deformation) numerical flux, the solver does not converge because the first-order information is meaningless in regions near the kink in the numerical flux, whereas the convergence is much faster and cleaner for the smoothed upwind flux (Figure 8).

\subsection{Time-dependent, inviscid Burgers' equation}

Next, we consider the time-dependent, inviscid Burgers' equation that governs nonlinear advection of a scalar quantity $U: \Omega \rightarrow \mathbb{R}$ through the space-time domain $\Omega:=\mathcal{T} \times \bar{\Omega}$,

$$
\frac{\partial U}{\partial t}+U \frac{\partial U}{\partial x}=0 \quad \text { in } \Omega, \quad U=U_{\infty} \quad \text { on } \Gamma_{i}(U)
$$


Initialization

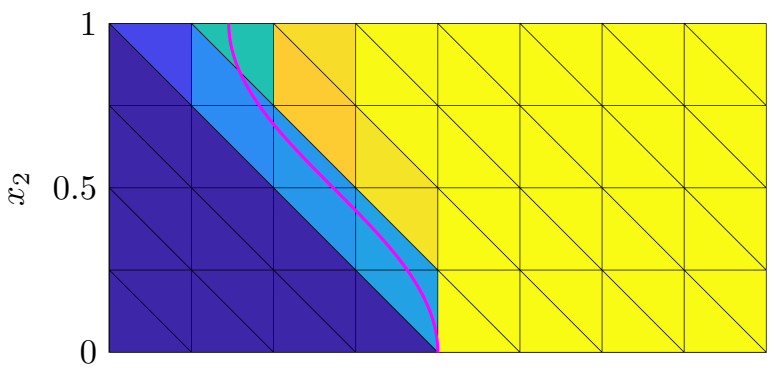

Converged $(q=2)$

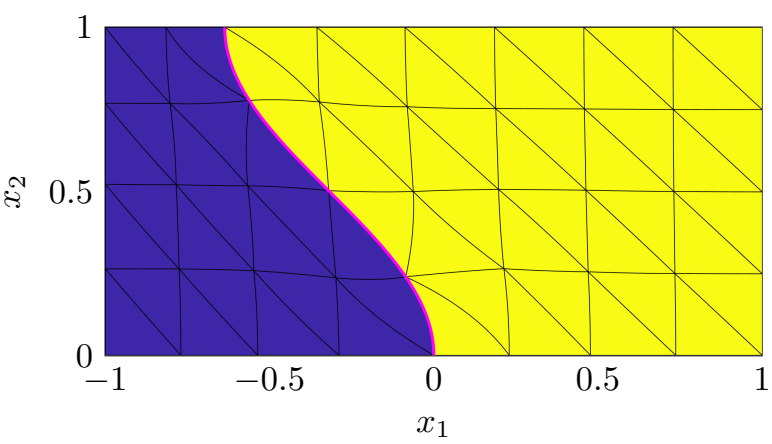

Converged $(q=1)$

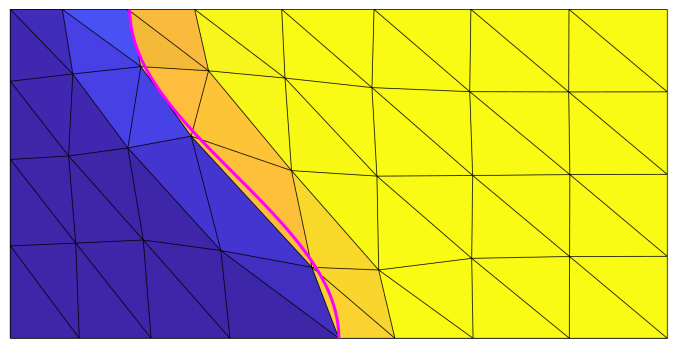

Converged $(q=3)$

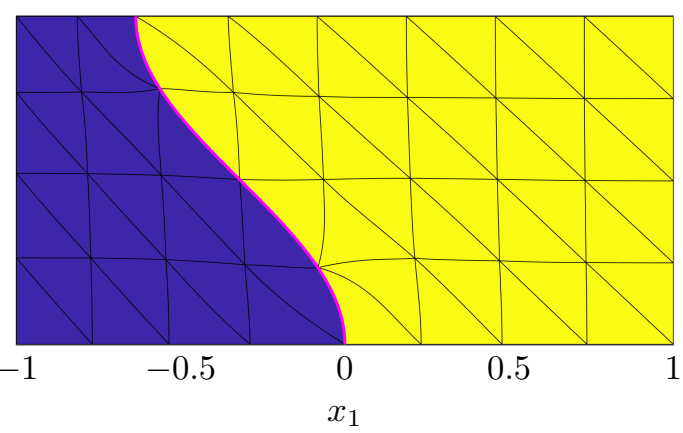

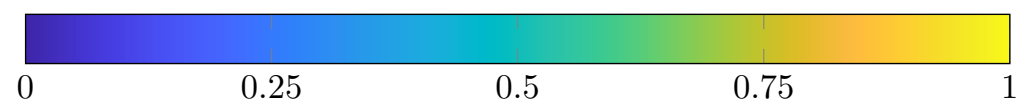

Figure 6: Solution of advection equation with the trigonometric shock using the using a $p=0$ basis for the solution and $q=1$ (top right), $q=2$ (bottom left), and $q=3$ (bottom right) basis for the mesh. The DG solution on a uniform triangular mesh with 64 elements is used to initialize the tracking method ( $t o p$ left). The magenta line highlights the discontinuity surface of the exact solution. The method converges to a mesh and solution that approximates the true solution as good as can be expected given the resolution of the finite element space; however, only the high-order elements provide a reasonable approximation on this coarse mesh. 

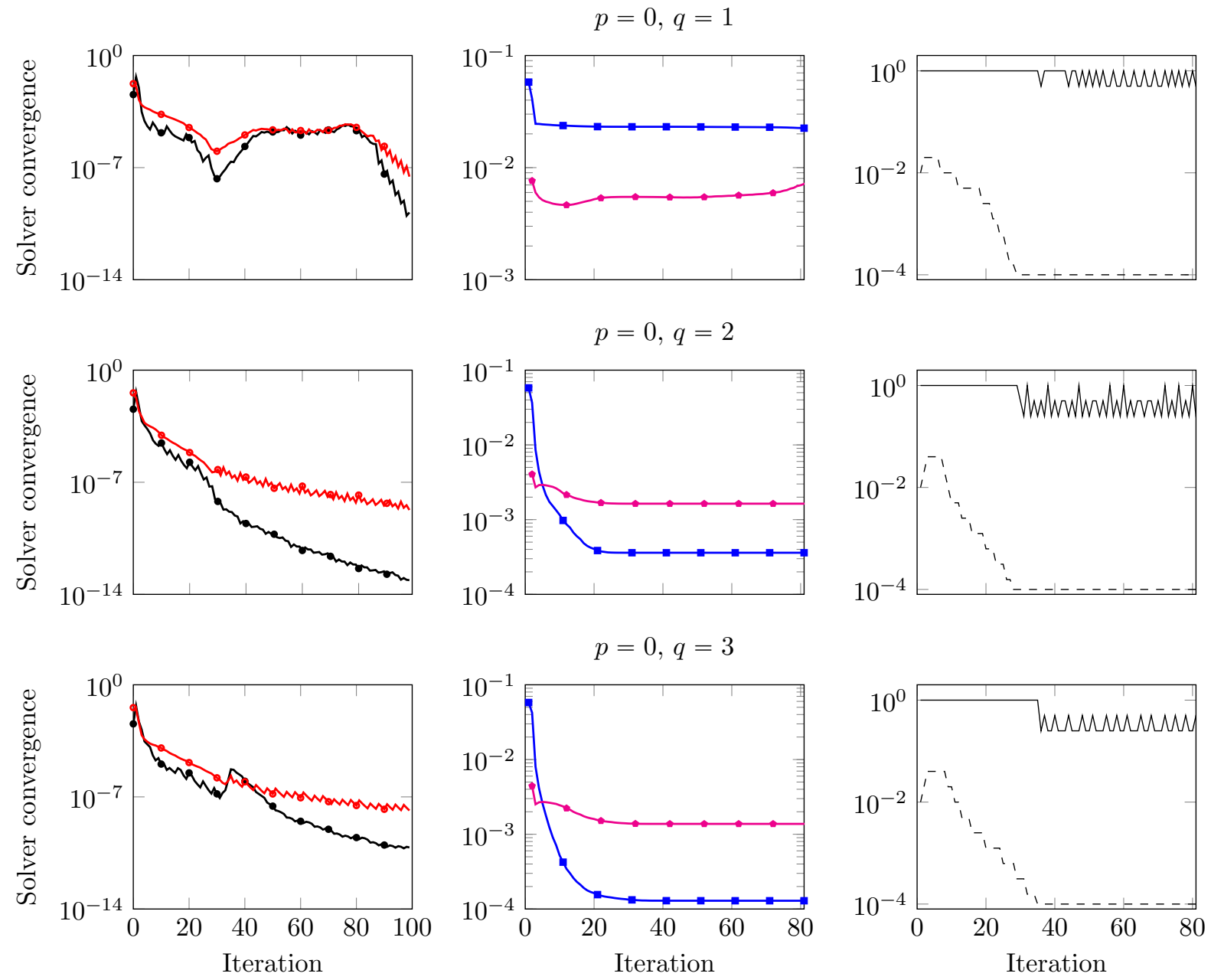

Figure 7: Convergence of the DG residual $\|\boldsymbol{r}(\boldsymbol{u}, \boldsymbol{x})\|(\rightarrow-)$, enriched DG residual $\|\boldsymbol{R}(\boldsymbol{u}, \boldsymbol{x})\|(\rightarrow-)$, optimality condition $\|\boldsymbol{c}(\boldsymbol{u}, \boldsymbol{x})\|(\longrightarrow)$, mesh distortion $\left\|\kappa \boldsymbol{R}_{\mathrm{msh}}(\boldsymbol{x})\right\|(\longrightarrow)$, and control of the regularization parameter $\left.(--)^{-}\right)$and step size $(\longrightarrow)$ for the tracking method applied to the advection equation with the trigonometric shock. Even though Newton convergence is not achieved, the proposed solver converges the KKT system to tight tolerances in a reasonable number of iterations.
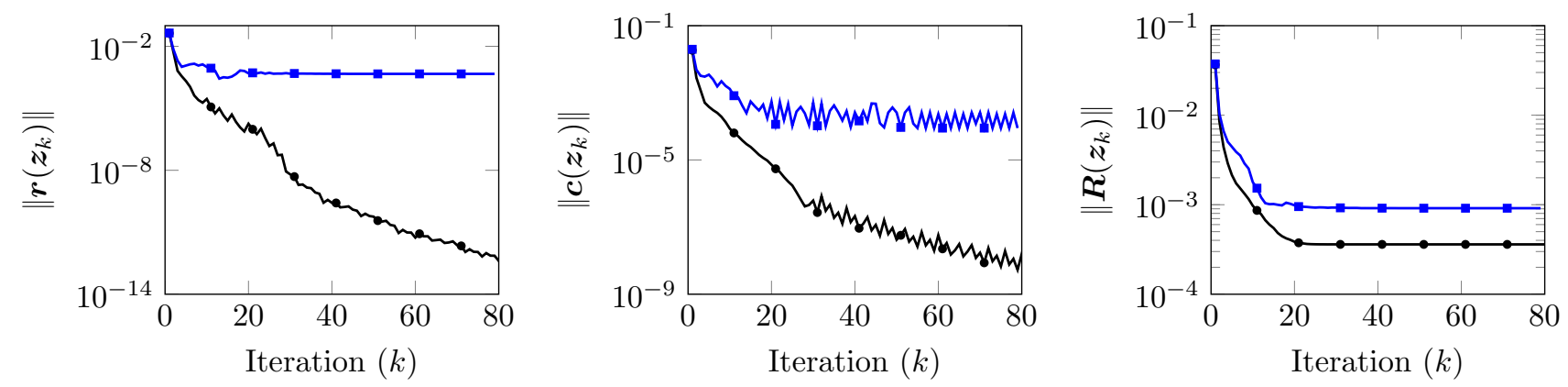

Figure 8: Convergence of the constraint (left), optimality condition (middle), and objective function (right) of the shock tracking optimization problem for linear advection with the trigonometric shock $(p=0, q=2)$ when the non-smooth upwind flux $\mathcal{H}_{\mathrm{up}}$ $(\longrightarrow)$ and smoothed $(a=10)$ upwind flux $\mathcal{H}_{\mathrm{up}}^{a}(\longrightarrow)$ are used as the numerical flux function. 
where $\mathcal{T}:=(0,1)$ is the time domain, $\bar{\Omega}:=(-1,1)$ is the one-dimensional spatial domain, $\Gamma_{i}(U):=\{(t, x) \in$ $\partial \Omega \mid \beta(U) \cdot n(x)<0\}$ is the inflow boundary, $\beta(U)$ is the space-time flow direction defined as

$$
\beta(U)=\left[\begin{array}{c}
1 \\
U
\end{array}\right]
$$

$n: \partial \Omega \rightarrow \mathbb{R}^{2}$ is the space-time unit outward normal, and $U_{\infty}: \partial \Omega \rightarrow \mathbb{R}$ is the inflow boundary condition. This fits the form of a general, steady conservation law over the space-time domain $\Omega$ with flux function

$$
F(U)=\left[\begin{array}{ll}
U & \frac{U^{2}}{2}
\end{array}\right]
$$

In the space-time setting, Burgers' equation has the same form as linear advection with the solutiondependent advection field (81). Similar to the advection equation, we consider a pure space-time upwind numerical flux since it satisfies conditions (i)-(iii) (Section 2.3)

$$
\mathcal{H}\left(U^{+}, U^{-}, n\right)=\mathcal{H}_{\text {up }}\left(U^{+}, U^{-}, n ; \beta\left(\frac{U^{+}+U^{-}}{2}\right)\right)
$$

and yields a stable DG discretization suitable for tracking. We also consider the smoothed version to satisfy condition (iv)

$$
\mathcal{H}\left(U^{+}, U^{-}, n\right)=\mathcal{H}_{\text {up }}^{a}\left(U^{+}, U^{-}, n ; \beta\left(\frac{U^{+}+U^{-}}{2}\right)\right) .
$$

We consider the following piecewise quadratic boundary condition

$$
U_{\infty}(t, x)=2(x+1)^{2}(1-H(x)),
$$

which is enforced via the pure upwind numerical flux evaluated at the interior state $U^{+}$and boundary state $U^{\partial}=U_{\infty}$.

For this problem, we consider a solution and mesh basis of equal polynomial degree $p=q$ up to $p=q=4$. The reference mesh is taken to be a uniform triangular mesh of the domain with 64 elements. The mesh and solution are initialized according to Section 5.1, including continuation in the polynomial degree. The SQP solver is used with $\lambda$ chosen adaptively (Section 4.6). The various DG/tracking parameters are set as follows: $\kappa=10^{-4}$ (mesh smoothing), smoothed upwind numerical flux $(a=10), \gamma_{0}=10^{-1}$ and $\gamma_{\min }=10^{-4}$ (regularization parameter adaptivity), and $N_{\max }=100, \epsilon_{1}=10^{-6}, \epsilon_{2}=10^{-10}$ (termination criteria). We initialize the $p=q=1$ simulation from the $p=1 \mathrm{DG}$ solution on the reference mesh. Even though the initial mesh is far from tracking the discontinuity (some faces are nearly orthogonal to the discontinuity, rather than parallel to it), our method tracks a faceted approximation to the discontinuity in only 40 iterations, requiring 7 element collapses (Figure 9).

The mesh and solution for the high-order elements $(p=q>1)$ are initialized from the $p=q=1$ tracking mesh and solution. These high-order approximations provide high-quality approximations of the discontinuous space-time solution on the coarse mesh (Figure 10). This can further be verified from the temporal slices (Figure 11), which show the moving discontinuity is perfectly tracked and the solution is smooth and non-oscillatory away from the discontinuity.

The convergence of the SQP solver in general is similar to convergence behavior observed with the linear advection equation with a trigonometric shock in that the higher the polynomial degree, the cleaner the convergence, and the enriched residual and mesh distortion converge to smaller absolute values, indicating a solution that provides a better approximation to the continuous weak form on a higher quality mesh (Figure 12). The line search is active at some intermediate iterations, but plays less of a role near convergence. Furthermore, the same observations regarding the tracking parameters made for the advection equation with the trigonometric shock hold for this problem: the smoothed upwind flux significantly improves convergence of the solver, and if $\kappa$ or $\gamma_{\min }$ are any smaller, too many elements will collapse onto the discontinuity surface for $p=q=1$. 


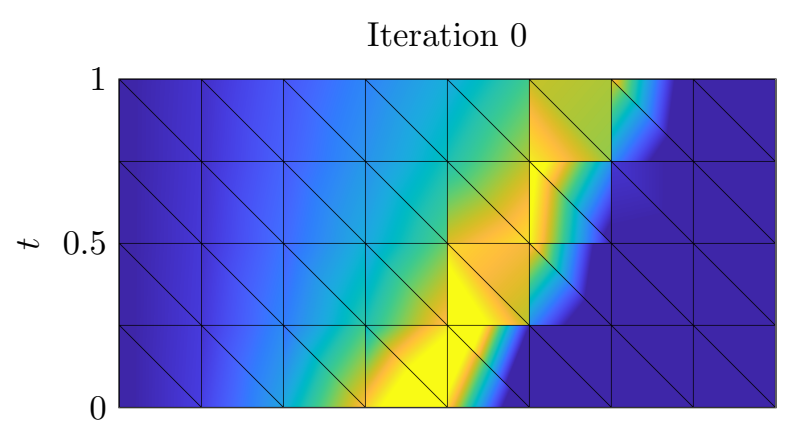

Iteration 30

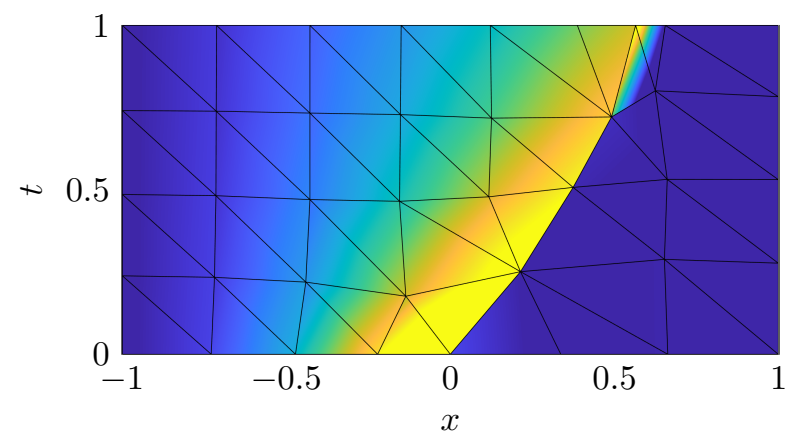

Iteration 20

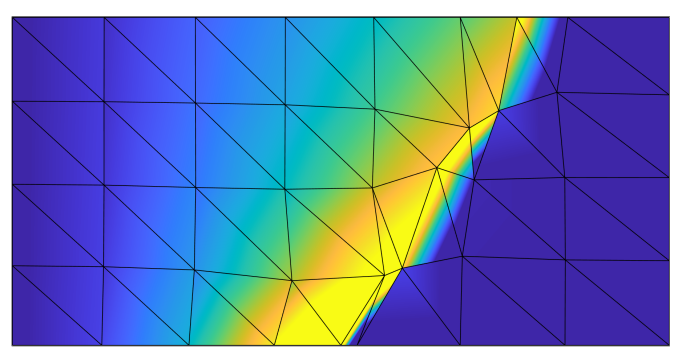

Iteration 40

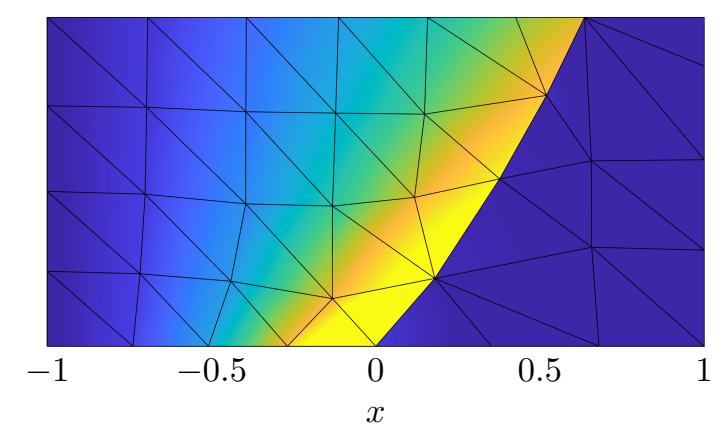

Figure 9: Space-time solution of one-dimensional, inviscid Burgers' equation using the tracking method at various iterations throughout the solution procedure using a $p=q=1$ basis for the solution and mesh. The method collapses 7 elements throughout the solution procedure and tracks a faceted approximation of the shock trajectory using the $q=1$ mesh in only 40 iterations.

\subsection{Steady, compressible Euler equations}

The Euler equations govern the steady flow of an inviscid, compressible fluid through a domain $\Omega \subset \mathbb{R}^{d}$

$$
\left(\rho v_{j}\right)_{, j}=0, \quad\left(\rho v_{i} v_{j}+p \delta_{i j}\right)_{, j}=0, \quad\left(\rho H v_{i}\right)_{, j}=0 \quad \text { in } \Omega
$$

where $\rho: \Omega \times(0, T) \rightarrow \mathbb{R}_{+}$is the density of the fluid, $v_{i}: \Omega \times(0, T) \rightarrow \mathbb{R}$ for $i=1, \ldots, d$ is the velocity of the fluid in the $i$ th coordinate direction, and $E: \Omega \times(0, T) \rightarrow \mathbb{R}_{+}$is the total energy of the fluid. The square velocity $q^{2}: \Omega \times(0, T) \rightarrow \mathbb{R}_{+}$and kinetic energy $k_{e}: \Omega \times(0, T) \rightarrow \mathbb{R}_{+}$of the fluid are

$$
q^{2}=v_{i} v_{i}, \quad k_{e}=\frac{1}{2} \rho q^{2} .
$$

The enthalpy of the fluid $H: \Omega \times(0, T) \rightarrow \mathbb{R}_{+}$is defined as

$$
\rho H=\rho E+P,
$$

where $P: \Omega \times(0, T) \rightarrow \mathbb{R}_{+}$is the pressure. For a calorically ideal fluid, the pressure and energy are related via the ideal gas law

$$
P=(\gamma-1)\left(\rho E-k_{e}\right)
$$

and the speed of sound is

$$
c=\sqrt{\gamma P / \rho} .
$$




$$
p=q=1
$$

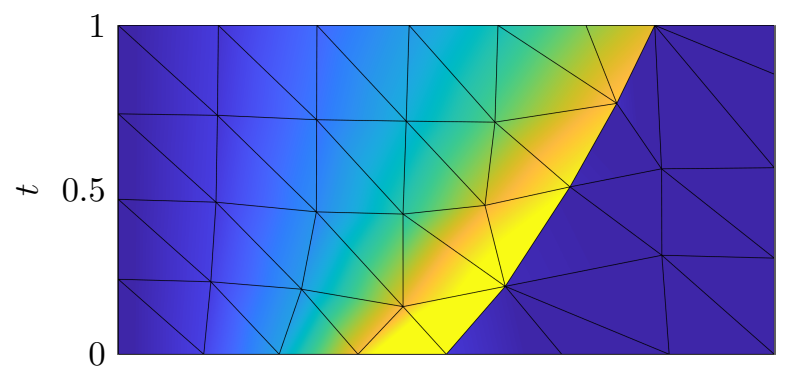

$$
p=q=2
$$

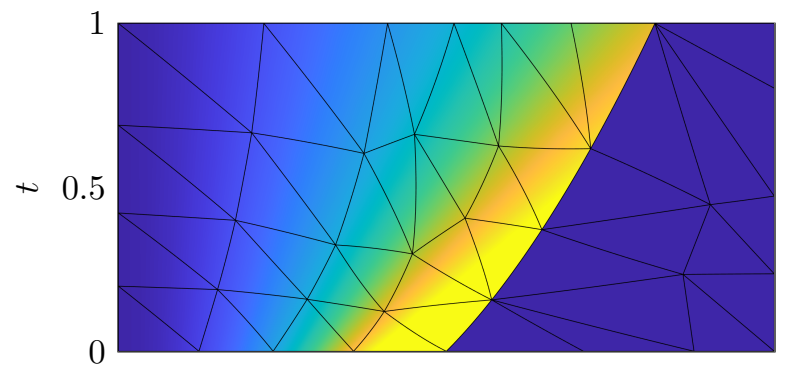

$$
p=q=3
$$

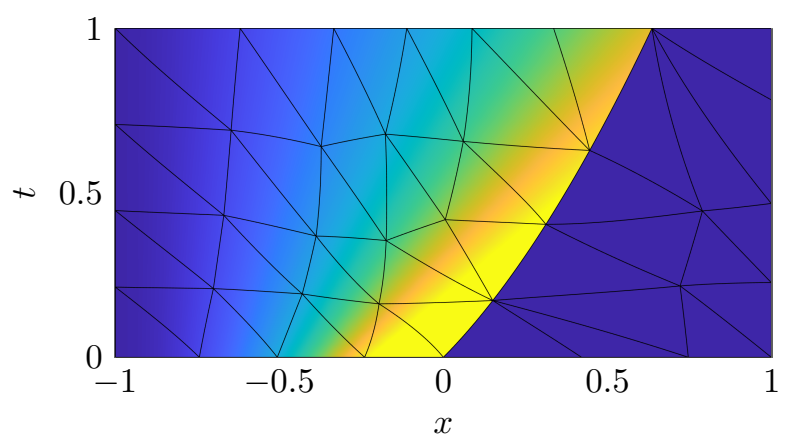

$p=q=1$

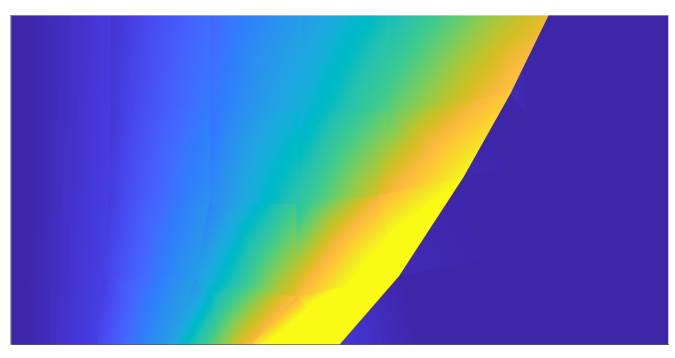

$$
p=q=2
$$

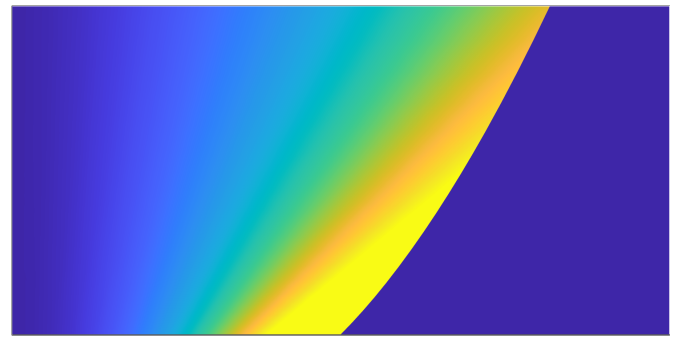

$$
p=q=3
$$

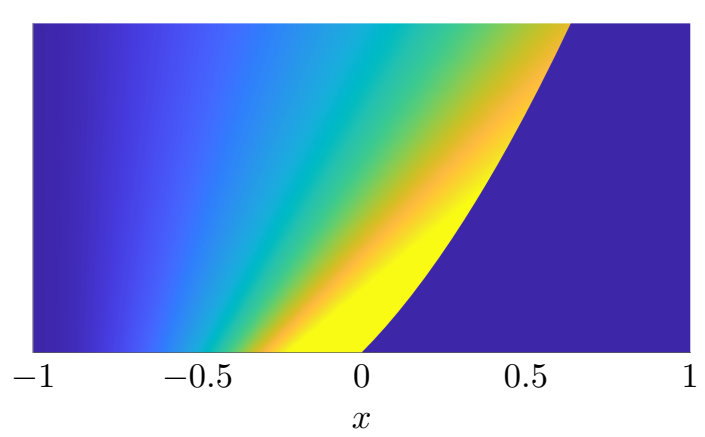

Figure 10: Space-time solution of one-dimensional, inviscid Burgers' equation using the proposed tracking method with a $p=q=1$ (top), $p=q=2$ (middle), and $p=q=3$ (bottom) basis for the solution and mesh with (left) and without (right) element boundaries. In all cases, the finite element solution provides a high-quality approximation to the true solution by tracking the discontinuity with a well-conditioned mesh. This is particularly true for the high-order elements that curve to the space-time trajectory of the discontinuity. 


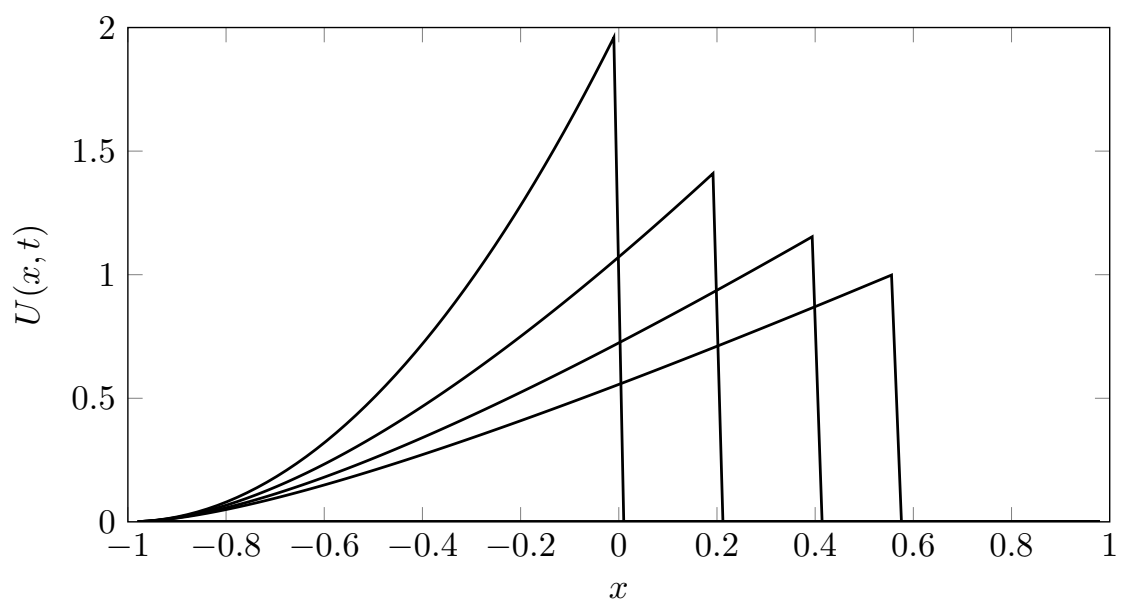

Figure 11: Temporal slices of the $p=q=4$ tracking solution to the inviscid Burgers' equation at times $t=0.05,0.35,0.65,0.95$.
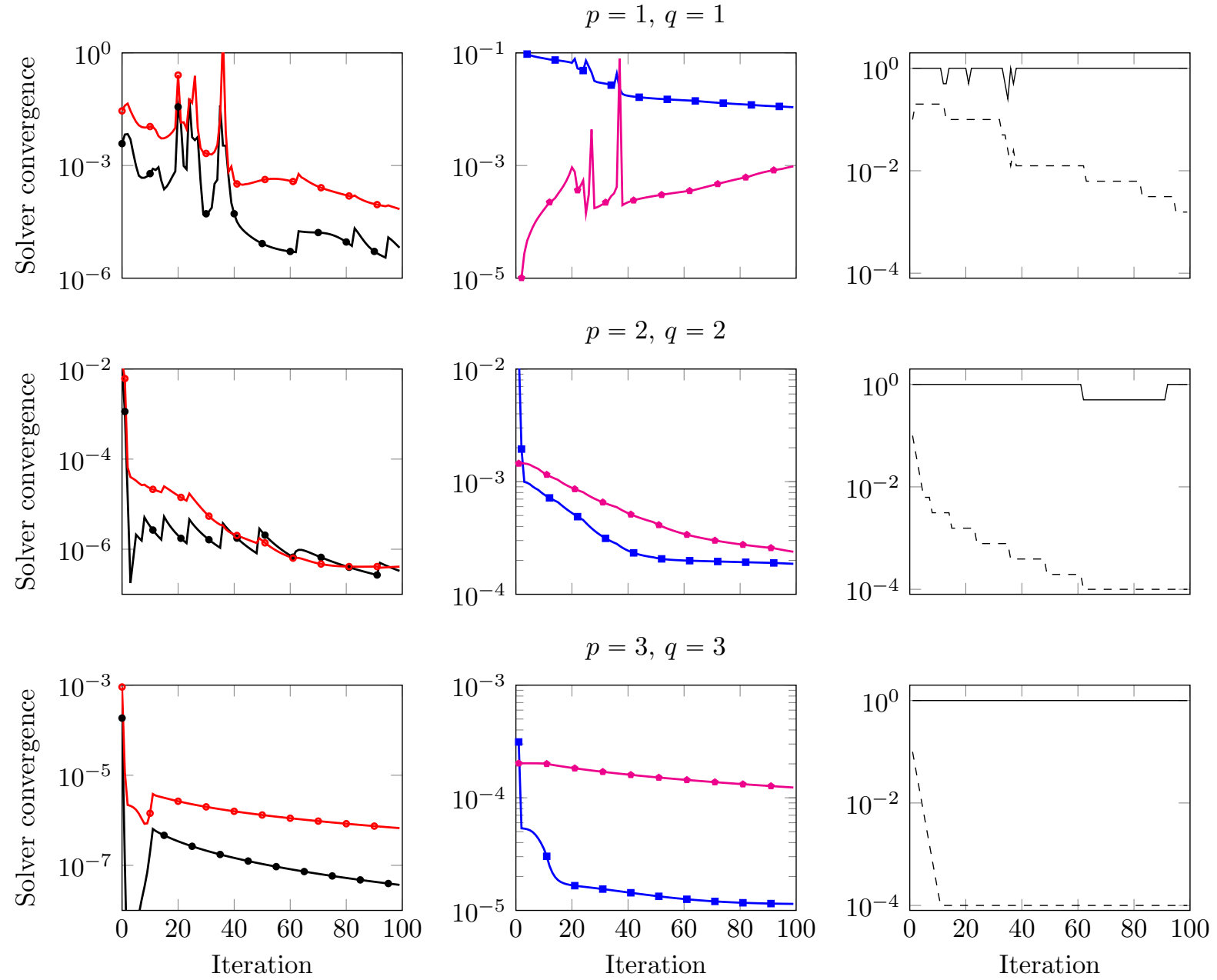

Figure 12: Convergence of the DG residual $\|\boldsymbol{r}(\boldsymbol{u}, \boldsymbol{x})\|(\rightarrow-)$, enriched DG residual $\|\boldsymbol{R}(\boldsymbol{u}, \boldsymbol{x})\|(\rightarrow-)$, optimality condition $\|\boldsymbol{c}(\boldsymbol{u}, \boldsymbol{x})\|(\longrightarrow)$, mesh distortion $\left\|\kappa \boldsymbol{R}_{\mathrm{msh}}(\boldsymbol{x})\right\|(\longrightarrow-)$ and control of the regularization parameter $\left(--^{-}\right)$and step size ( - ) for the tracking method applied to the inviscid Burgers' equation. 
The density, velocity, and energy are combined into a vector of conservative variables $U$ and the Euler equations take the form of an inviscid conservation law (1) with flux function $F(U)$

$$
U=\left[\begin{array}{c}
\rho \\
\rho v \\
\rho E
\end{array}\right], \quad F(U)=\left[\begin{array}{c}
\rho v^{T} \\
\rho v v^{T}+P I_{2} \\
\rho H v^{T}
\end{array}\right],
$$

where $I_{2}$ is the $2 \times 2$ identity matrix.

We use Roe's flux [32] as the numerical flux function to ensure a stable discretization suitable for tracking since it satisfies (i)-(iii) (Section 2.3); however, it is not smooth with respect to variations in the domain deformation. To eliminate entropy violating rarefaction shocks and improve the smoothness with respect to the domain deformations, we modify the interior numerical fluxes with the Harten-Hyman entropy fix [17]. This leads to a numerical flux function that violates (iii), as observed in [8], but recovers smoothness with respect to mesh deformation, which proves to be a reasonable trade-off.

We consider three types of boundaries: slip wall $\left(\Gamma_{w}\right)$, supersonic inflow $\left(\Gamma_{D}\right)$, and supersonic outflow $\left(\Gamma_{N}\right)$. The supersonic inflow is also known as a farfield or Dirichlet condition and the supersonic outflow is a Neumann condition. For a slip wall $(v \cdot n=0)$, the boundary state is defined as

$$
U_{w}^{\partial}(U, n):=\left[\begin{array}{c}
\rho \\
\rho v^{-} \\
\rho E
\end{array}\right]
$$

where $v^{-}=(v-2 v \cdot n) n$ is the velocity reflected about the normal. For a supersonic inflow, all characteristics are coming into the domain and the boundary state depends solely on the prescribed density $\rho_{\infty}$, velocity $v_{\infty}$, and pressure $p_{\infty}$

$$
U_{D}^{\partial}(U):=\left[\begin{array}{c}
\rho_{\infty} \\
\rho_{\infty} v_{\infty} \\
\frac{p_{\infty}}{\gamma-1}+\frac{\rho_{\infty}}{2} v_{\infty} \cdot v_{\infty}
\end{array}\right] .
$$

Finally, at a supersonic outflow, all characteristics are leaving the domain and the boundary state is taken from the interior

$$
U_{N}^{\partial}(U):=U
$$

All boundary conditions are enforced via the pure Roe flux (without entropy fix) evaluated at the interior state $U^{+}$and appropriate boundary state

$$
U^{\partial}(U, n)= \begin{cases}U_{w}^{\partial}(U, n) & \text { on } \Gamma_{w} \\ U_{D}^{\partial}(U) & \text { on } \Gamma_{D} \\ U_{N}^{\partial}(U) & \text { on } \Gamma_{N} .\end{cases}
$$

A useful property of the inviscid flows is the enthalpy is constant throughout the domain. Therefore, to quantify the error in the numerical solution obtained using the proposed method, we will use the deviation of the flow enthalpy from the inflow enthalpy, $H_{\infty}:=\frac{\gamma}{\gamma-1} \frac{p_{\infty}}{\rho_{\infty}}+\frac{1}{2} v_{\infty} \cdot v_{\infty}$

$$
e_{H}(U):=\sqrt{\frac{\int_{\Omega}\left(H(U)-H_{\infty}\right)^{2}}{\int_{\Omega} d V}} .
$$

\subsubsection{Supersonic flow over wedge}

First we consider supersonic flow $\left(M_{\infty}=2\right)$ over a $\theta=10^{\circ}$ inclined plane (Figure 13). Since all wall boundaries are straight-sided and the incoming flow is uniform, the flow is piecewise constant and the shocks are straight. 


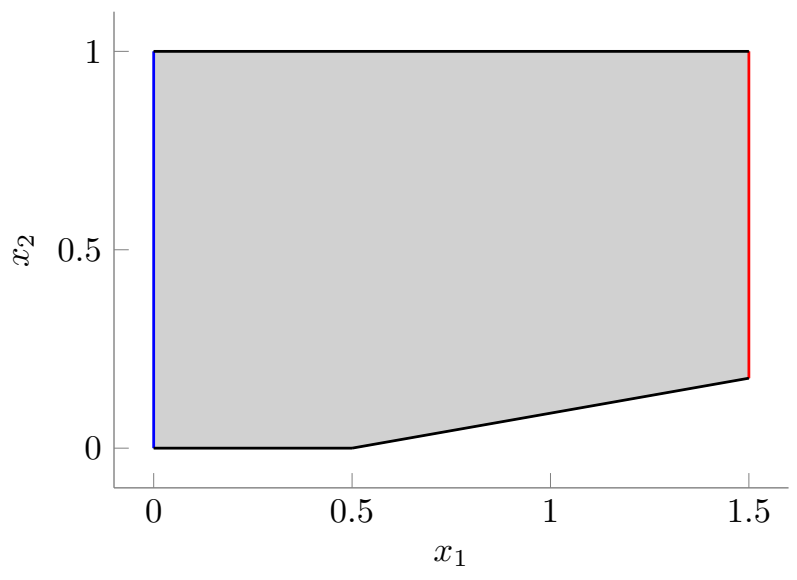

Figure 13: Geometry and boundary conditions of the wedge problem. Boundary conditions: slip wall ( $\longrightarrow$ ), supersonic inflow with $\rho_{\infty}=1.4, v_{\infty}=(2,0), p_{\infty}=1\left(M_{\infty}=2\right)(-)$, and supersonic outflow $(-)$.

Therefore, we apply the tracking method with a $p=0$ polynomial basis for the solution and $q=1$ basis for the mesh. The reference mesh is taken to be a uniform triangular mesh of the domain with 48 elements. The solver is initialized with the $p=0$ DG solution on the reference mesh (Section 5.1). The SQP solver is used with $\lambda$ chosen adaptively (Section 4.6). The various DG/tracking parameters are set as follows: $\kappa=0$ (no mesh smoothing), Roe flux (without entropy fix), $\gamma_{0}=1$ and $\gamma_{\text {min }}=10^{-8}$ (regularization parameter adaptivity), and $\epsilon_{1}=10^{-8}$, and $\epsilon_{2}=10^{-12}$ (termination criteria). After only 20 iterations, the mesh perfectly tracks the shock (Figure 14) and the DG solution closely matches the exact solution; the enthalpy error of the solution is $e_{H}=7.94 \times 10^{-10}$. The solver performs similarly to the advection equation with a straight discontinuity and therefore a full discussion is omitted for brevity.

\subsubsection{Supersonic flow over airfoil}

Next, we apply the proposed tracking method to solve for supersonic flow over a NACA0012 airfoil (Figure 15). This is a difficult problem because there are two distinct shocks that must be resolved: a bow shock ahead of the leading edge and an oblique shock off the tail. The reference mesh is taken as an unstructured triangular mesh of the domain with 160 elements generated by DistMesh [30]. The mesh and solution are initialized according to Section 5.1, including continuation in the polynomial degree. The SQP solver is used with $\lambda$ chosen adaptively (Section 4.6). The various DG/tracking parameters are set as follows: $\kappa=10^{-4}$ (mesh smoothing), Roe flux with entropy fix, $\gamma_{0}=1$ and $\gamma_{\min }=1$ (regularization parameter adaptivity), and $N_{\max }=100, \epsilon_{1}=10^{-6}, \epsilon_{2}=10^{-10}$ (termination criteria).

For the $p=q=1$ simulation, the proposed method tracks a faceted approximation to the discontinuity in only 100 iterations, requiring 11 element collapses and maintain high-quality elements, despite the coarse elements in the initial mesh that do not conform to either shock (Figure 16). Even though the discontinuities are successfully tracked, the solution is under-resolved, particularly near the body, since the large $p=1$ elements are not sufficient to resolve the solution in this region. As a result, the enthalpy error is large $e_{H}=1.30 \times 10^{-3}$. The $p=q=2$ and $p=q=3$ tracking solutions provide highly accurate approximations to the true flow even on this coarse mesh due to the high-order resolution of the discontinuity surface with curved elements and high-order approximation of the flow (Figure 16); the enthalpy error for the $p=q=2$ tracking solution is $e_{H}=6.73 \times 10^{-5}$ and for $p=q=3$ is $e_{H}=1.02 \times 10^{-5}$. The perfect (zero-thickness) capturing of the shocks and high-order approximation of the solution can further be seen from the solution slices in Figure 17.

For this problem, the convergence of the solver is not as clean as the other problems (Figure 18) and heavily relies on the line search throughout the solution procedure to ensure sufficient decrease in the $\ell_{1}$ merit function. However, the solver still drives the KKT conditions to reasonable tolerances within 100 iterations for the $p=q=3$ simulation. The spikes in the KKT residuals around iterations 20 and 60 in the $p=q=1$ simulation are due to element collapses. Our choice of algorithmic parameters were critical for this case. If $\kappa$ is significantly larger, the element collapses would not occur and the mesh would not be able to track the shocks, particularly the weaker shock at the trailing edge. Furthermore, it was important to 


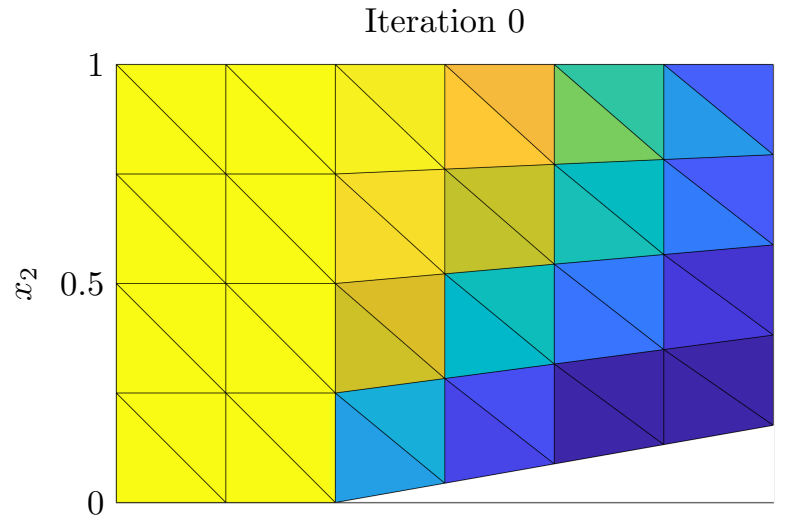

Iteration 8

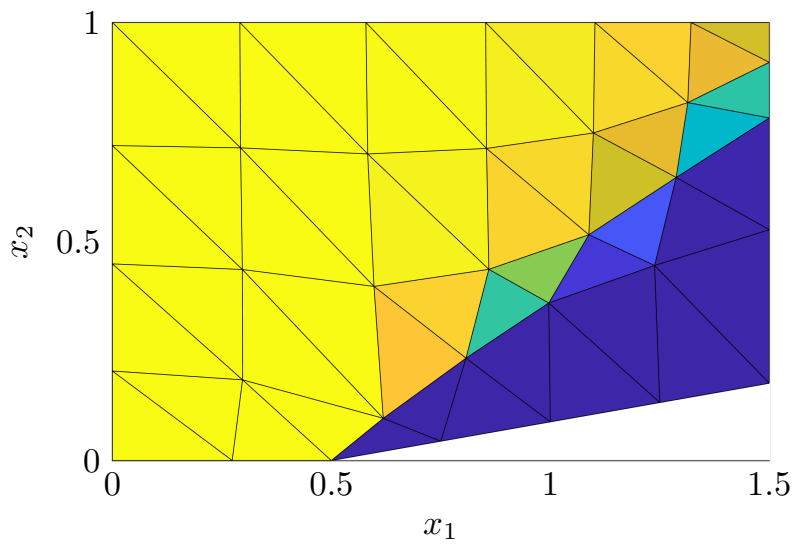

Iteration 4

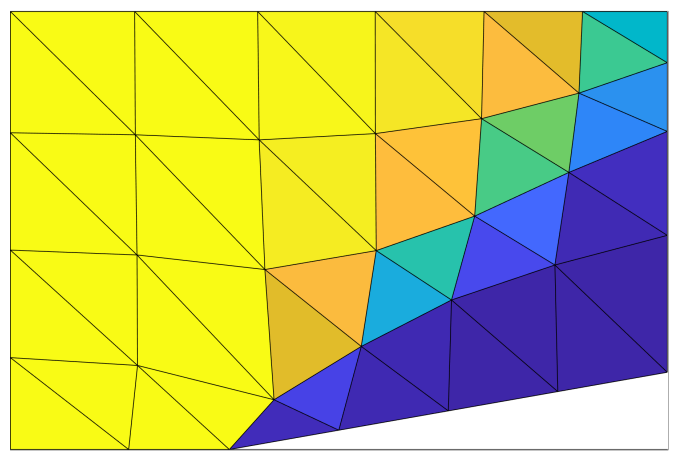

Iteration 20

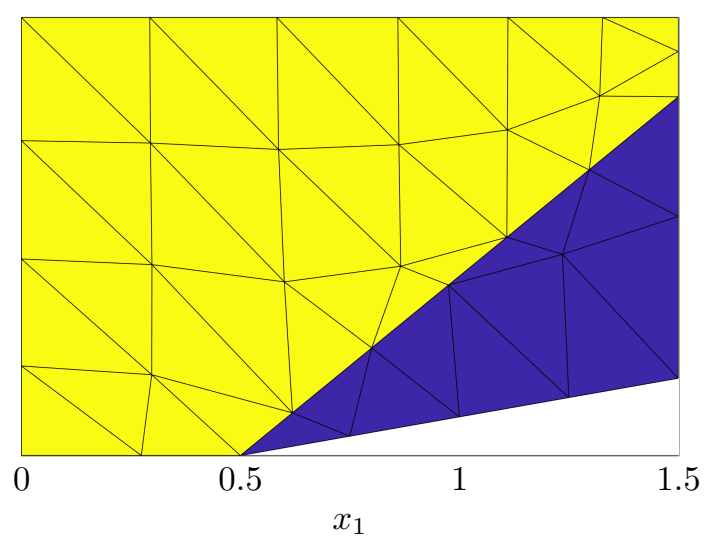

1.65

1.74

1.83

1.91

Figure 14: Solution (Mach) of Euler equations over a wedge (supersonic regime) using the tracking method at various iterations throughout the solution procedure using $p=0$ basis for the solution and $q=1$ basis for the mesh. The method converges to nearly the exact solution (enthalpy error $e_{H}=7.94 \times 10^{-10}$ ), in only 20 iterations.

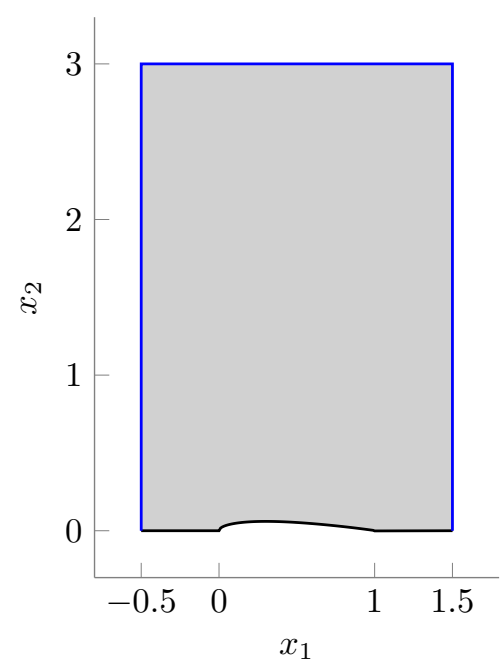

Figure 15: Geometry and boundary conditions of the airfoil problem. Boundary conditions: slip wall $(-)$ and farfield (characteristic) conditions with $\rho_{\infty}=1.4, v_{\infty}=\left(M_{\infty}, 0\right), p_{\infty}=1\left(M_{\infty}=0.85\right.$ for the transonic case and $M_{\infty}=1.5$ for the supersonic case). 


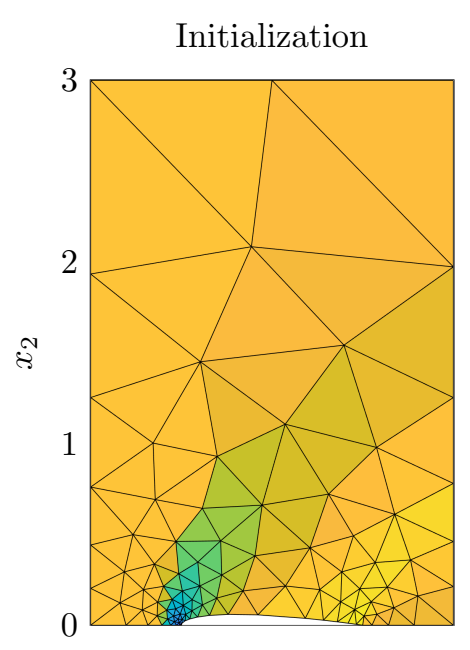

Converged $(p=1)$
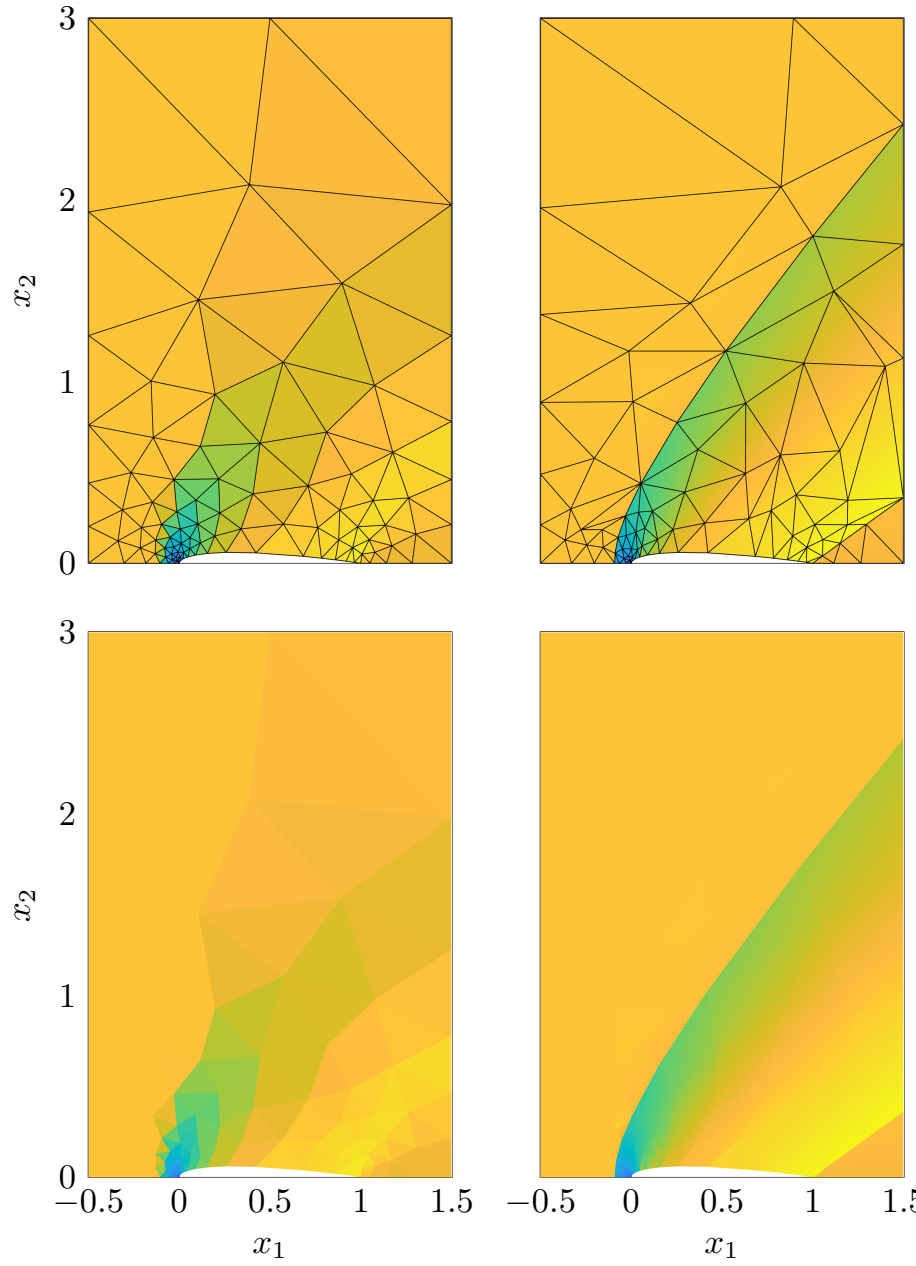

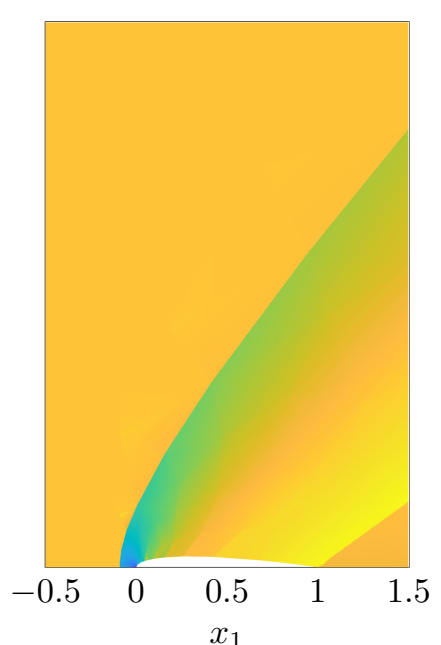

$x_{1}$
Converged $(p=2)$
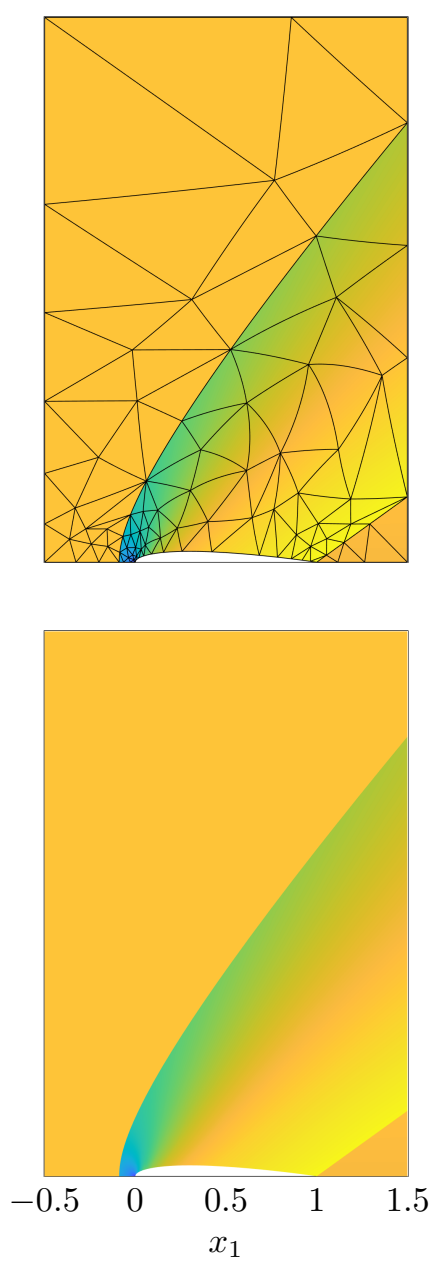

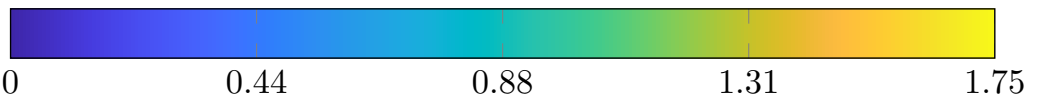

Figure 16: Solution (Mach) of Euler equations over the NACA0012 airfoil $\left(M_{\infty}=1.5\right)$ using the proposed tracking method with a $p=q=1$ (center) and $p=q=2$ (right) basis for the solution and mesh with (top) and without (bottom) element boundaries. In both cases, the tracking procedure successfully tracks the shocks given the resolution in the finite element space, despite the initial mesh and solution (left) being far from aligned with the shock. The high-order $(p=q=2)$ basis yields an accurate approximation to the flow on the coarse mesh while the low-order $(p=q=1)$ solution is under-resolved. 

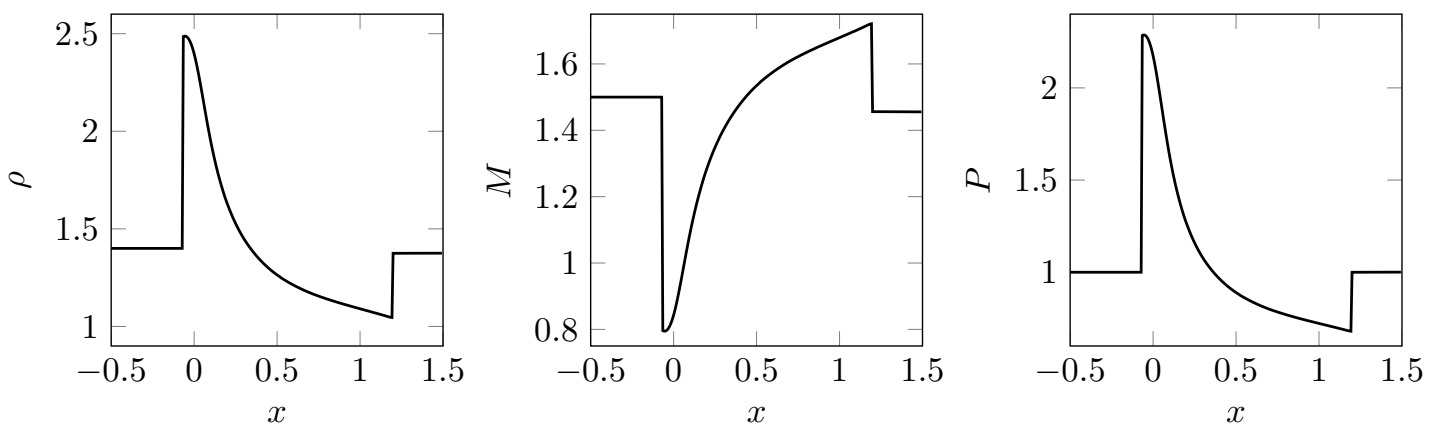

Figure 17: Slices of density (left), Mach number (center), and pressure (right) of the $p=q=3$ tracking solution along the curve $\Gamma:=\{(s, 0.14) \mid s \in(-0.5,1.5)\}$ for the NACA problem. The discontinuities are captured perfectly between DG elements and the solution is smooth and non-oscillatory away from the discontinuities, indicating that the solution is well-resolved and the discontinuities are successfully tracked.

use the relatively large value of $\gamma_{\min }=1$, otherwise the line search would be taxed more heavily in the later iterations and completely stalled convergence.

Finally, we mention that this is the first problem where our choice of the elliptic regularization matrix with the coefficients chosen inversely proportional to the size of the element in the reference domain (Section 4.5) is significant. The other choices we explored included the identity matrix [8], elliptic PDE stiffness matrix without volume-based weighting [11], and other choices involving the finite element mass matrix. All of these options performed similarly in creating unacceptably large search direction in regions near the leading and trailing edge (small elements) relative to other regions in the mesh (large elements). Without a line search, these steps would cause the mesh to entangle and the simulation to crash. With the line search, the step size in regions with larger elements would be driven nearly to zero causing the solver to fail to track the discontinuity in these regions. From this, we conclude the combination of the elliptic PDE stiffness matrix and the weighting of the coefficients inversely with respect to the element size in the reference domain is important for meshes with elements of significantly varying size.

\subsubsection{Transonic flow over airfoil}

Next, we consider transonic flow $\left(M_{\infty}=0.85\right)$ over the same NACA0012 airfoil and domain from the previous section (Figure 15). This problem has a shock attached to the curved airfoil profile, which requires a nontrivial boundary mapping (Section 3.3) to ensure nodes slide along the airfoil surface. We construct the mapping $\chi$ using the procedure in [40], i.e., the $x$-coordinates of the nodes on the surface are taken as optimization parameters and the $y$-coordinates are determined from the expression for the airfoil profile

$$
y(x)=0.6\left(0.2960 \sqrt{x}-0.126 x-0.3516 x^{2}+0.2843 x^{3}-0.1036 x^{4}\right) .
$$

The reference mesh is taken as an unstructured triangular mesh of the domain with 127 elements generated by DistMesh [30]. The mesh and solution are initialized according to Section 5.1, including continuation in the polynomial degree. The SQP solver is used with $\lambda$ chosen adaptively (Section 4.6). The various DG/tracking parameters are set as follows: $\kappa=10^{-3}$ (mesh smoothing), Roe flux with entropy fix, $\gamma_{0}=10^{-2}$ and $\gamma_{\min }=1$ (regularization parameter adaptivity), and $N_{\max }=30, \epsilon_{1}=10^{-6}, \epsilon_{2}=10^{-10}$ (termination criteria).

For polynomial degrees $p=q=1,2,3$, the tracking method accurately tracks the attached discontinuity without requiring element collapses and produces an accurate flow solution given the resolution of the approximation space (Figure 19). The high resolution of the $p=q=3$ simulation can be seen through the slices of the solution (Figure 20): the discontinuity is captured perfectly and the solution away from the discontinuity is smooth and non-oscillatory. The convergence of the method has been thoroughly studied in the previous sections and omitted for brevity. 

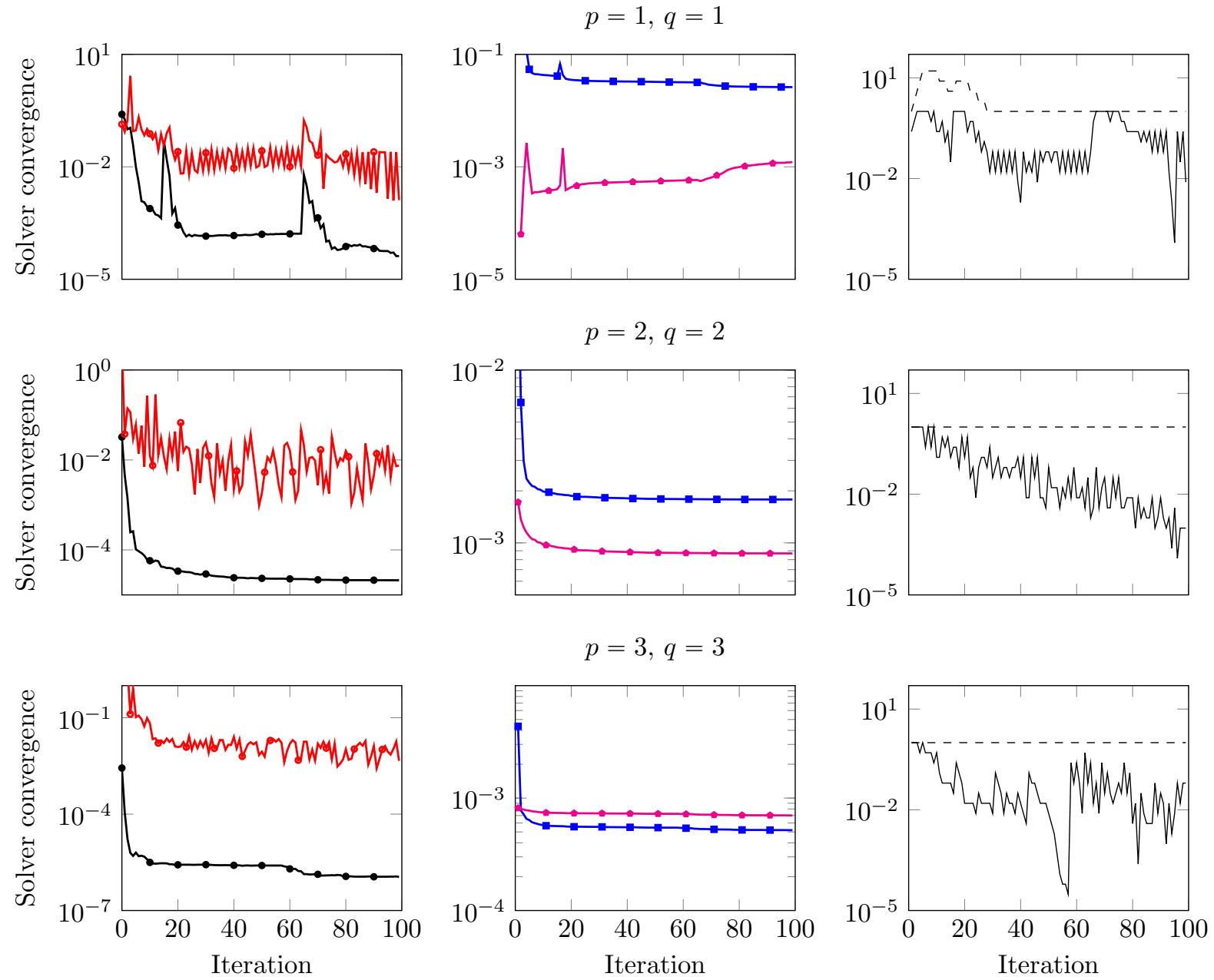

Figure 18: Convergence of the DG residual $\|\boldsymbol{r}(\boldsymbol{u}, \boldsymbol{x})\|(\rightarrow-)$, enriched DG residual $\|\boldsymbol{R}(\boldsymbol{u}, \boldsymbol{x})\|(\rightarrow-)$, optimality condition $\|\boldsymbol{c}(\boldsymbol{u}, \boldsymbol{x})\|(\longrightarrow)$, mesh distortion $\left\|\kappa \boldsymbol{R}_{\mathrm{msh}}(\boldsymbol{x})\right\|(--)$ and control of the regularization parameter $\left.(--)^{-}\right)$and step size $(-)$ for the tracking method applied to solve the supersonic flow around the NACA airfoil. For this difficult problem, the convergence of the KKT system is not as clean as the other problems and relies heavily on the line search; however, the solver still tracks the shocks and returns an accurate flow as seen from the solution plots (Figures 16-17). The spikes in the KKT conditions for $p=q=1$ are due to element collapses. 


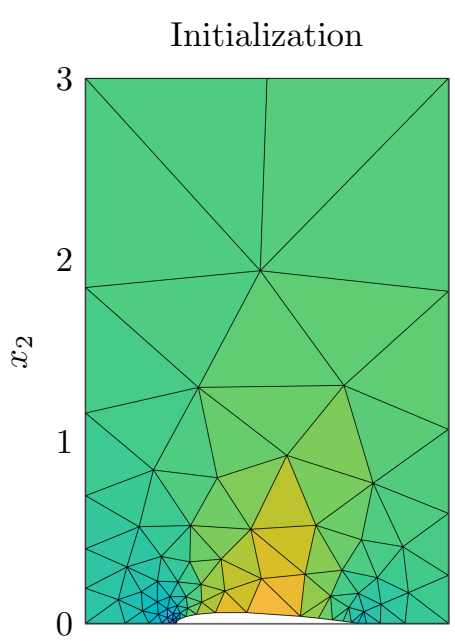

Converged $(p=1)$
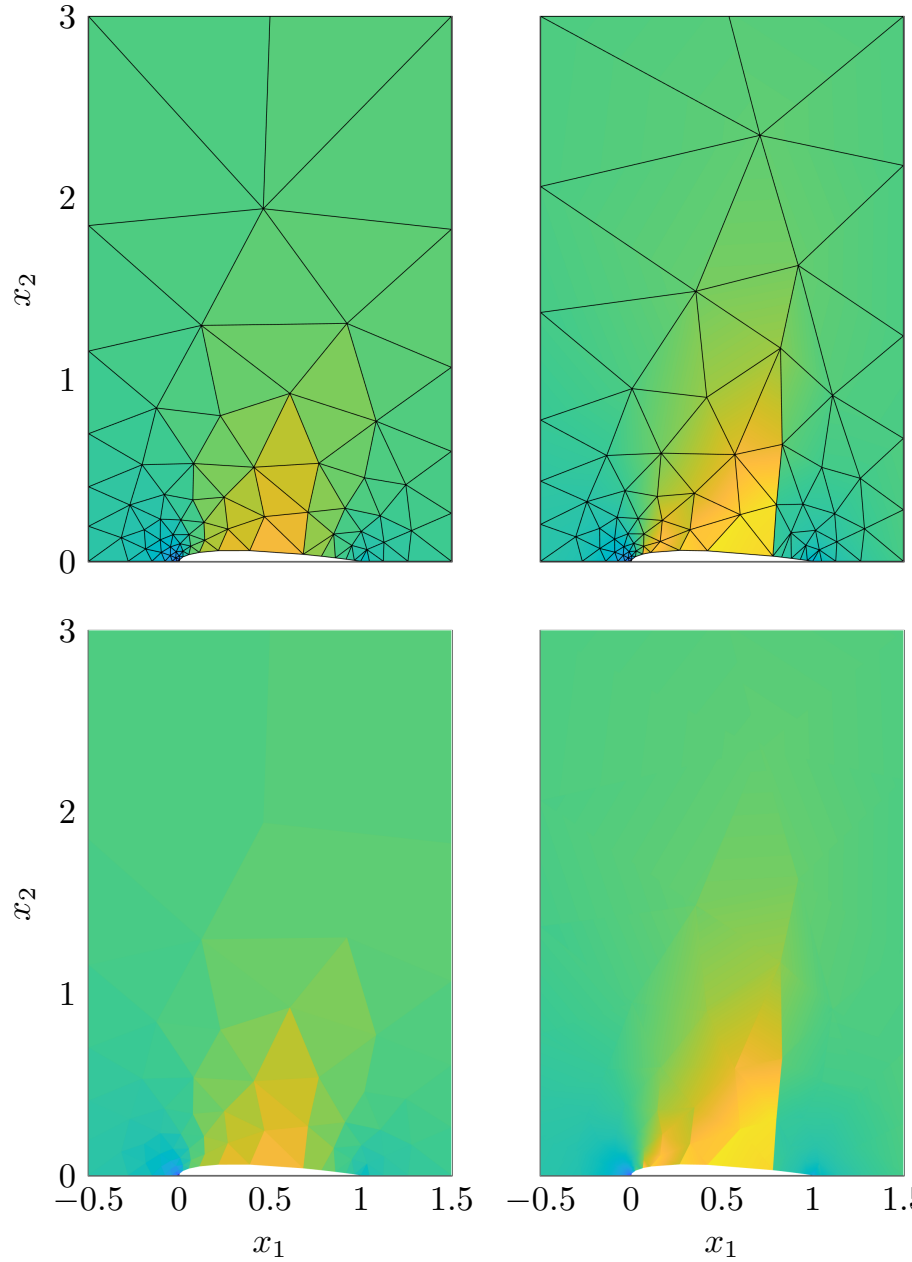

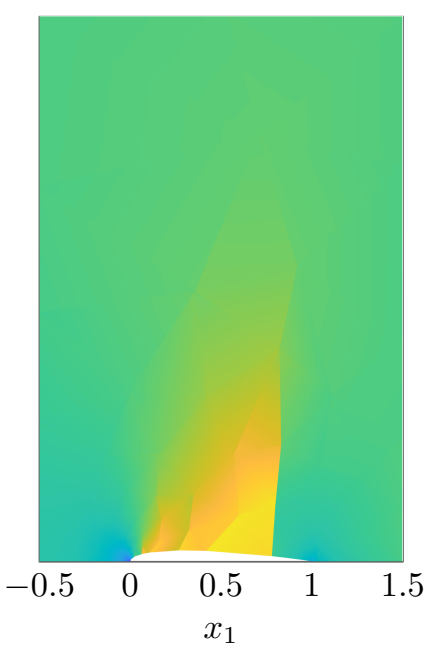

Converged $(p=2)$
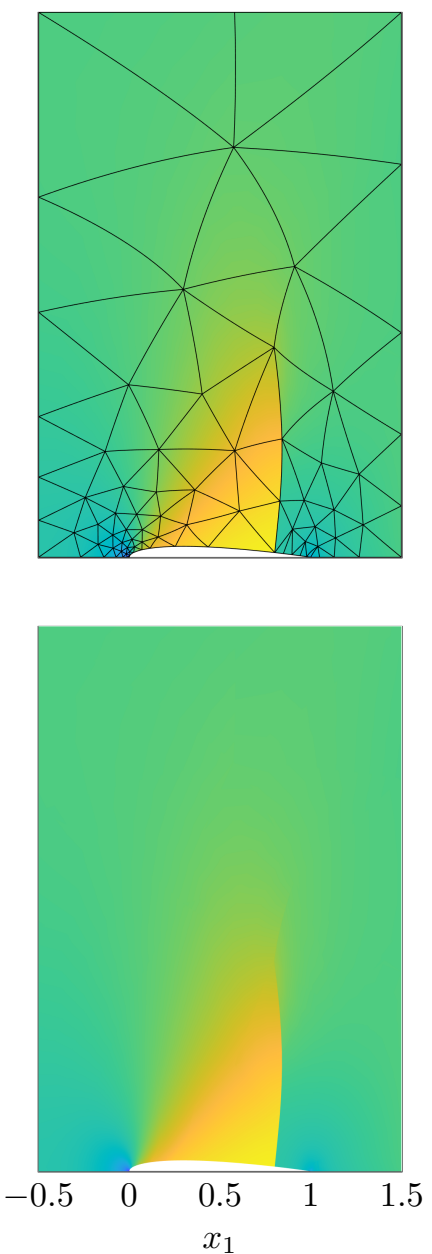

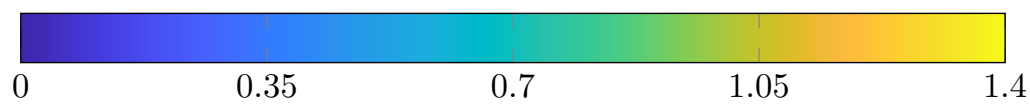

Figure 19: Solution (Mach) of Euler equations over the NACA0012 airfoil $\left(M_{\infty}=0.85\right)$ using the proposed tracking method with a $p=q=1$ (center) and $p=q=2$ (right) basis for the solution and mesh with (top) and without (bottom) element boundaries. In both cases, the tracking procedure successfully tracks the shocks given the resolution in the finite element space, despite the initial mesh and solution (left) being far from aligned with the shock.
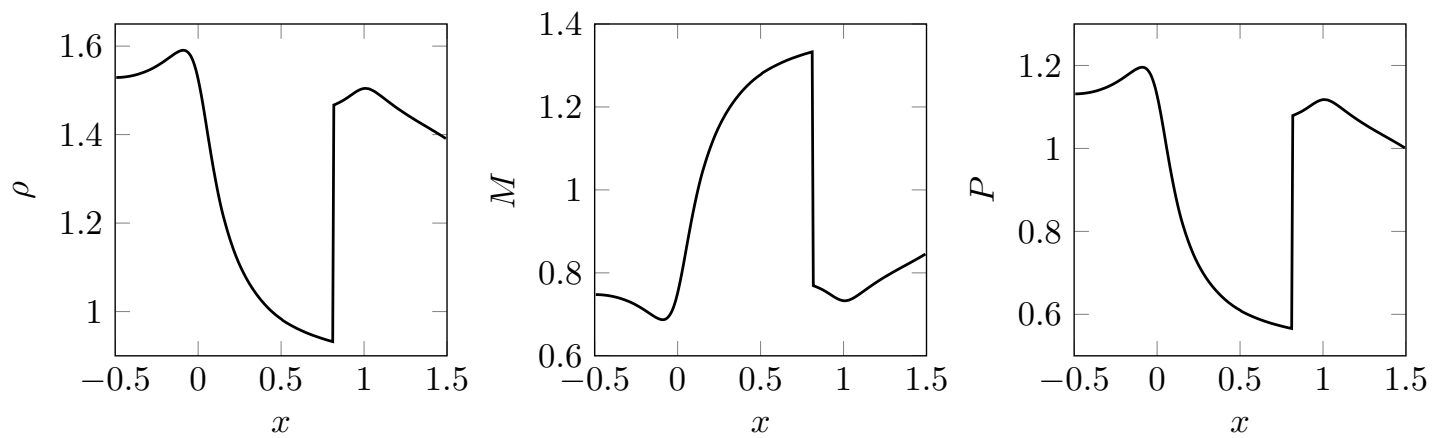

Figure 20: Slices of density (left), Mach number (center), and pressure (right) of the $p=q=3$ tracking solution along the curve $\Gamma:=\{(s, 0.14) \mid s \in(-0.5,1.5)\}$ for the NACA problem $\left(M_{\infty}=0.85\right)$. The discontinuity is captured perfectly between DG elements and the solution is smooth and non-oscillatory away from the discontinuity, indicating that the solution is well-resolved. 


\section{Conclusions}

We introduced an improved formulation of the optimization-based implicit shock tracking method proposed in [39] and an associated solver that leverages the structure of the problem. The proposed optimization problem minimizes the DG residual in an enriched test and the distortion of the mesh, constrained by the standard DG residual (equal trial and test spaces). The enriched residual is a practical surrogate for the violation of the weak formulation of the conservation law; its magnitude serves an error indicator for a DG solution. Therefore, penalizing the enriched DG residual promotes alignment of the element faces with discontinuities; otherwise, the DG solution would oscillate about the discontinuities and provide a poor approximation to the conservation law. The proposed solver for the constrained optimization problem over the DG solution and mesh coordinates is an SQP method that uses a Levenberg-Marquardt Hessian approximation and is globalized via a line search on the $\ell_{1}$ merit function. The Hessian approximation is regularized with the stiffness matrix corresponding to a linear elliptic partial equation with coefficients inversely proportional to the local element size and adaptively chosen regularization parameter, which turns out to be significant for meshes containing elements of significantly different size.

For problems where the finite element space contains the exact solution, the SQP method exhibits Newtonlike convergence to the exact solution, which was demonstrated via linear advection of a scalar through a constant advection field and supersonic, inviscid flow over a wedge. The framework was also shown to be effective in accurately resolving more complex flows with intricate shock structures using coarse, high-order meshes as demonstrated using the inviscid Burgers' equation (time-dependent), and transonic and supersonic flow over a NACA0012 airfoil. For these problems, the convergence of the solver is slower, but still drives the first-order optimality conditions to tight tolerances in a reasonable number of iterations.

Future work will develop iterative solvers and preconditioners for the SQP linear system in (57) to make the approach practical for large-scale problems. We also intend to further improve the robustness of the solver by incorporating pseudo-transient continuation to avoid the need to initialize from a $p=0$ DG solution and use continuation on the polynomial degrees $p$ (DG solution) and $q$ (domain deformation). We will also develop a method of lines and adaptive space-time approaches to handle more complex time-dependent cases where a single space-time discretization may not be feasible. Finally, we will demonstrate high-order convergence of the method for inviscid flows in two- and three-dimensions and consider more complex flows, including viscous and relevant 3D problems.

\section{Acknowledgments}

This work was supported in part by the Director, Office of Science, Office of Advanced Scientific Computing Research, U.S. Department of Energy under Contract No. DE-AC02-05CH11231. The content of this publication does not necessarily reflect the position or policy of any of these supporters, and no official endorsement should be inferred.

\section{References}

[1] Douglas N Arnold, Franco Brezzi, Bernardo Cockburn, and L Donatella Marini. Unified analysis of discontinuous Galerkin methods for elliptic problems. SIAM Journal on Numerical Analysis, 39(5):1749$1779,2002$.

[2] M. J. Baines, S. J. Leary, and M. E. Hubbard. Multidimensional least squares fluctuation distribution schemes with adaptive mesh movement for steady hyperbolic equations. SIAM Journal on Scientific Computing, 23(5):1485-1502, 2002.

[3] C. E. Baumann and J. T. Oden. A discontinuous $h p$ finite element method for the Euler and NavierStokes equations. Int. J. Numer. Methods Fluids, 31(1):79-95, 1999. Tenth International Conference on Finite Elements in Fluids (Tucson, AZ, 1998).

[4] JB Bell, GR Shubin, and JM Solomon. Fully implicit shock tracking. Journal of Computational Physics, 48(2):223-245, 1982. 
[5] Paul Boggs and Jon Tolle. Sequential quadratic programming for large-scale nonlinear optimization. Journal of Computational and Applied Mathematics, 124(1):123-137, 2000.

[6] A. Burbeau, P. Sagaut, and Ch.-H. Bruneau. A problem-independent limiter for high-order Runge-Kutta discontinuous Galerkin methods. J. Comput. Phys., 169(1):111-150, 2001.

[7] Bernardo Cockburn and Chi-Wang Shu. Runge-Kutta discontinuous Galerkin methods for convectiondominated problems. J. Sci. Comput., 16(3):173-261, 2001.

[8] Andrew Corrigan, Andrew Kercher, and David Kessler. A moving discontinuous Galerkin finite element method for flows with interfaces. International Journal for Numerical Methods in Fluids, 89(9):362-406, 2019.

[9] Andrew Corrigan, Andrew Kercher, and David Kessler. The moving discontinuous Galerkin method with interface condition enforcement for unsteady three-dimensional flows. In AIAA Scitech 2019 Forum, AIAA-2019-0642.

[10] Andrew Corrigan, Andrew Kercher, David Kessler, and Devon Wood-Thomas. Application of the moving discontinuous Galerkin method with interface condition enforcement to shocked compressible flows. In 2018 Fluid Dynamics Conference, AIAA-2018-4272.

[11] Andrew Corrigan, Andrew Kercher, David Kessler, and Devon Wood-Thomas. Convergence of the moving discontinuous galerkin method with interface condition enforcement in the presence of an attached curved shock. In AIAA Aviation 2019 Forum, AIAA-2019-3207.

[12] Alain Dervieux, David Leservoisier, Paul-Louis George, and Yves Coudière. About theoretical and practical impact of mesh adaptation on approximation of functions and PDE solutions. Internat. J. Numer. Methods Fluids, 43(5):507-516, 2003. ECCOMAS Computational Fluid Dynamics Conference, Part I (Swansea, 2001).

[13] Krzysztof J Fidkowski. Output error estimation strategies for discontinuous Galerkin discretizations of unsteady convection-dominated flows. International Journal for Numerical Methods in Engineering, 88(12):1297-1322, 2011.

[14] A. Gargallo-Peiró, X. Roca, J. Peraire, and J. Sarrate. A distortion measure to validate and generate curved high-order meshes on CAD surfaces with independence of parameterization. Internat. J. Numer. Methods Engrg., 106(13):1100-1130, 2016.

[15] James Glimm, Xiaolin Li, Yingjie Liu, Zhiliang Xu, and Ning Zhao. Conservative front tracking with improved accuracy. SIAM Journal on Numerical Analysis, 41(5):1926-1947, 2003.

[16] Ami Harten, Björn Engquist, Stanley Osher, and Sukumar R. Chakravarthy. Uniformly high-order accurate essentially nonoscillatory schemes. III. J. Comput. Phys., 71(2):231-303, 1987.

[17] Ami Harten and James M Hyman. Self adjusting grid methods for one-dimensional hyperbolic conservation laws. Journal of computational Physics, 50(2):235-269, 1983.

[18] J. S. Hesthaven and T. Warburton. Nodal discontinuous Galerkin methods, volume 54 of Texts in Applied Mathematics. Springer, New York, 2008. Algorithms, analysis, and applications.

[19] Jan Hesthaven and Tim Warburton. Nodal Discontinuous Galerkin Methods: Algorithms, Analysis, and Applications. Springer Science \& Business Media, 2007.

[20] Guang-Shan Jiang and Chi-Wang Shu. Efficient implementation of weighted ENO schemes. J. Comput. Phys., 126(1):202-228, 1996.

[21] Car Kelley and David Keyes. Convergence analysis of pseudo-transient continuation. SIAM Journal on Numerical Analysis, 35(2):508-523, 1998. 
[22] Andrew Kercher, Andrew Corrigan, and David Kessler. The Moving Discontinuous Galerkin method with Interface Condition Enforcement for viscous flows. In AIAA Scitech 2020 Forum, AIAA-2020-1315.

[23] Patrick M. Knupp. Algebraic mesh quality metrics. SIAM Journal on Scientific Computing, 23(1):193218, 2001.

[24] Xu-Dong Liu, Stanley Osher, and Tony Chan. Weighted essentially non-oscillatory schemes. J. Comput. Phys., 115(1):200-212, 1994.

[25] Rainald Löhner. Applied Computational Fluid Dynamics Techniques: An Introduction Based on Finite Element Methods. John Wiley \& Sons, 2008.

[26] Andrew Majda. Compressible fluid flow and systems of conservation laws in several space variables, volume 53. Springer Science \& Business Media, 2012.

[27] Jorge Nocedal and S Wright. Numerical optimization, series in operations research and financial engineering. Springer, 2006.

[28] Jayandran Palaniappan, Scott T Miller, and Robert B Haber. Sub-cell shock capturing and spacetime discontinuity tracking for nonlinear conservation laws. International Journal for Numerical Methods in Fluids, 57(9):1115-1135, 2008.

[29] P.-O. Persson and J. Peraire. Sub-cell shock capturing for discontinuous Galerkin methods. In 44th AIAA Aerospace Sciences Meeting and Exhibit, Reno, Nevada, 2006. AIAA-2006-0112.

[30] Per-Olof Persson and Gilbert Strang. A simple mesh generator in MATLAB. SIAM review, 46(2):329$345,2004$.

[31] Philip Roe and Hiroaki Nishikawa. Adaptive grid generation by minimizing residuals. International Journal for Numerical Methods in Fluids, 40(1-2):121-136, 2002.

[32] Philip L Roe. Approximate Riemann solvers, parameter vectors, and difference schemes. Journal of computational physics, 43(2):357-372, 1981.

[33] GR Shubin, AB Stephens, and HM Glaz. Steady shock tracking and Newton's method applied to one-dimensional duct flow. Journal of Computational Physics, 39(2):364-374, 1981.

[34] Greg R Shubin, AB Stephens, HM Glaz, AB Wardlaw, and LB Hackerman. Steady shock tracking, Newton's method, and the supersonic blunt body problem. SIAM Journal on Scientific and Statistical Computing, 3(2):127-144, 1982.

[35] F Taghaddosi, WG Habashi, G Guevremont, and D Ait-Ali-Yahia. An adaptive least-squares method for the compressible euler equations. International Journal for Numerical Methods in Fluids, 31(7):11211139, 1999.

[36] Eleuterio Toro, Michael Spruce, and William Speares. Restoration of the contact surface in the HLLRiemann solver. Shock Waves, 4(1):25-34, 1994.

[37] J.-Y. Trepanier, M. Paraschivoiu, M. Reggio, and R. Camarero. A conservative shock fitting method on unstructured grids. Journal of Computational Physics, 126(2):421 - 433, 1996.

[38] John Van Rosendale. Floating shock fitting via lagrangian adaptive meshes. Technical Report ICASE Report No. 94-89, Institute for Computer Applications in Science and Engineering, 1994.

[39] Matthew Zahr and Per-Olof Persson. An optimization-based approach for high-order accurate discretization of conservation laws with discontinuous solutions. Journal of Computational Physics, 365:105-134, 2018.

[40] Matthew J. Zahr and Per-Olof Persson. An $r$-adaptive, high-order discontinuous Galerkin method for flows with attached shocks. In AIAA Scitech 2020 Forum, AIAA-2020-0523.

[41] Xiaolin Zhong. High-order finite-difference schemes for numerical simulation of hypersonic boundarylayer transition. J. Comput. Phys., 144(2):662-709, 1998. 